\title{
Mesons in strong magnetic fields: (I) General analyses
}

\author{
Koichi Hattori ${ }^{\mathrm{a}, \mathrm{b}}$, Toru Kojo ${ }^{\mathrm{c}, \mathrm{d}}$, Nan $\mathrm{Su}^{\mathrm{e}}$ \\ ${ }^{a}$ RIKEN BNL Research Center, Brookhaven National Laboratory, Upton NY 11973, USA \\ ${ }^{b}$ Theoretical Research Division, Nishina Center, RIKEN, Wako, Saitama 351-0198, Japan \\ ${ }^{c}$ Key Laboratory of Quark and Lepton Physics (MOE) and Institute of Particle Physics, Central China Normal \\ University, Wuhan 430079, China \\ ${ }^{d}$ Department of Physics, University of Illinois at Urbana-Champaign, $1110 \mathrm{~W}$. Green Street, Urbana, Illinois \\ 61801, USA \\ ${ }^{e}$ Institut für Theoretische Physik, Goethe-Universität Frankfurt, 60438 Frankfurt am Main, Germany
}

\begin{abstract}
We study properties of neutral and charged mesons in strong magnetic fields $|e B| \gg \Lambda_{\mathrm{QCD}}^{2}$ with $\Lambda_{\mathrm{QCD}}$ being the QCD renormalization scale. Assuming long-range interactions, we examine magnetic-field dependences of various quantities such as the constituent quark mass, chiral condensate, meson spectra, and meson wavefunctions by analyzing the Schwinger-Dyson and Bethe-Salpeter equations. Based on the density of states obtained from these analyses, we extend the hadron resonance gas (HRG) model to investigate thermodynamics at large $B$. As $B$ increases the meson energy behaves as a slowly growing function of the meson's transverse momenta, and thus a large number of meson states is accommodated in the low energy domain; the density of states at low temperature is proportional to $B^{2}$. This extended transverse phase space in the infrared regime significantly enhances the HRG pressure at finite temperature, so that the system reaches the percolation or chiral restoration regime at lower temperature compared to the case without a magnetic field; this simple picture would offer a gauge invariant and intuitive explanation of the inverse magnetic catalysis.
\end{abstract}

\section{Introduction}

In uniform magnetic fields, a trajectory of a charged particle wraps around a magnetic flux, leading to the discretized orbital levels known as the Landau levels. Each level has degenerate states and the density of states is given by $|e B| / 2 \pi$. Also, spin- $\frac{1}{2}$ fermions are subject to the Zeeman energy splitting, with which the $n$-th Landau level $(n \mathrm{LL})$ has the following spectrum at

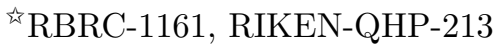


the tree level,

$$
E_{n}\left(p_{3}\right)=\sqrt{p_{3}^{2}+2 n|e B|+m^{2}},
$$

where the magnetic fields are applied to the $x_{3}$-direction, and $e$ and $m$ are the electric coupling constant and the fermion mass, respectively. A remarkable aspect for the fermionic case is that the energy of the lowest Landau level (LLL) at $n=0$ does not depend on the value of $B$, since the energy gained by the Zeeman effect cancels out the zero point energy arising from the orbital motion. Therefore the B-dependence of the LLL energy appears only through the radiative corrections. Those radiative corrections vary for different theories: our objective is to use such sensitivity to explore the structure of theories, especially Quantum Chromodynamics in the infrared (IR) regime. The characteristic scale is given by the QCD renormalization scale parameter, $\Lambda_{\mathrm{QCD}} \sim 0.2 \mathrm{GeV}$. (For an extensive review on magnetized systems, see Ref. [1].)

The magnetic field we shall consider is strong, $|e B| \gg \Lambda_{\mathrm{QCD}}^{2}$, perhaps much stronger than can be realized in experimental laboratories ${ }^{1}$; the magnetic fields at RHIC and LHC are $|e B| \sim$ $0.04 \mathrm{GeV}^{2}$ and $\sim 0.3 \mathrm{GeV}^{2}$, respectively, and persist only for a short time $[2,3]$. Thus the applicability of our arguments to phenomenology is subtle, but it is not our primary purpose. The strong field regime has been studied in the lattice simulations [5, 6, 7, 8, 9], and we use them to test various theoretical concepts and methods of calculations, aiming at applications to other unexplored domain, e.g., physics of cold and dense QCD. Indeed, the similarities between the low energy dynamics of QCD at high density and in strong magnetic fields have been pointed out $[10,11,12]$. For example, the "QCD Kondo effect", that is induced by the infrared instability near the Fermi surface, was recently studied first in dense QCD [13], and then it was shown that the similarity between the Fermi surface effect and the $(1+1)$ dimensional low energy dynamics in strong magnetic fields manifests itself as the "magnetically induced QCD Kondo effect" [14].

Specific problems address in the present work are theoretical paradoxes observed in the recent lattice QCD studies. Two problems can be thought of as the representatives: (i) The B-dependence of the chiral condensate [5]: The chiral effective models or chiral perturbation theory confirmed the magnetic catalysis [15] and explain the lattice data at $|e B| \ll \Lambda_{\mathrm{QCD}}^{2}$ well, while beyond $|e B| \sim 0.1-0.3 \mathrm{GeV}^{2}$, their predictions start to deviate from the lattice results which show the linear rising behavior, $\langle\bar{\psi} \psi\rangle \sim|e B| \Lambda_{\mathrm{QCD}}$. (ii) Inverse magnetic catalysis [6, 7]: The (2+1)-flavor lattice results at the physical pion mass show that the chiral restoration temperature $\left(T_{c}\right)$ decreases

\footnotetext{
${ }^{1}$ Beyond laboratory experiments, larger magnetic fields may be achieved. At the stage of electroweak (EW) transition in the early universe, $|e B|$ could reach $\sim 1 \mathrm{GeV}^{2}$ [4], but this size is much smaller than other scales relevant at early epoch. Magnetars have surface magnetic fields of electron mass scale and might have even larger magnetic fields at their cores by squeezing the surface fluxes, but how magnetic fluxes are distributed in magnetars remains an unsettled issue.
} 
at larger $B$, by $10-20 \%$ at $|e B| \sim 1 \mathrm{GeV}^{2}$, and this decreasing behavior continues to the maximal magnetic fields achieved on the lattice, $|e B| \simeq 3.3 \mathrm{GeV}^{2}[16]$. Typical model calculations [17] instead predict the qualitatively opposite behavior: $T_{c}$ grows rather rapidly as $B$ increases.

Effective models leading to these paradoxes have certain common features. Those models predict the dynamically generated quark mass, which is large $\left(\gg \Lambda_{\mathrm{QCD}}\right)$ and strongly $B$-dependent, e.g., $M \sim|e B|^{1 / 2}$ or $\sim \Lambda_{\mathrm{UV}} \mathrm{e}^{-c /|B|}$ ( $\Lambda_{\mathrm{UV}}$ is the UV cutoff of the model). Let us see how this estimate causes the aforementioned problems. At large $B$, the proper fermion bases are the Ritus bases by using which one can include the background $B$ effects in the full order. The chiral condensate can be written as $\left[\int_{p_{L}} \equiv \int \mathrm{d}^{2} p_{L} /(2 \pi)^{2}, p_{L}=\left(p_{0}, p_{3}\right)\right]$

$$
\langle\bar{\psi} \psi\rangle_{4 \mathrm{D}}=\frac{|e B|}{2 \pi}\langle\bar{\psi} \psi\rangle_{2 \mathrm{D}}, \quad\langle\bar{\psi} \psi\rangle_{2 \mathrm{D}} \equiv-\int_{p_{L}} \operatorname{tr}\left[S_{\mathrm{LLL}}^{2 \mathrm{D}}\left(p_{L}\right)+\sum_{n=1} S_{n \mathrm{LL}}^{2 \mathrm{D}}\left(p_{L}\right)\right]
$$

where $S_{n L L}^{2 \mathrm{D}}$ is the two-dimensional propagator for the $n$-th LL. Therefore if the mass gap rapidly grows as $B$ increases, the two-dimensional condensate $\langle\bar{\psi} \psi\rangle_{2 \mathrm{D}}$ also does. Then $\langle\bar{\psi} \psi\rangle_{4 \mathrm{D}}$ no longer depends on $B$ linearly, contradicting with the lattice results. The estimate of $M \gg \Lambda_{\mathrm{QCD}}$ also causes a problem for the critical temperature, because with such large mass gap the thermal fluctuations of quarks are not activated until the temperature reaches $T \gg \Lambda_{\mathrm{QCD}}$. Then the chiral restoration temperature keeps growing as $B$ increases, contradicting with the inverse magnetic catalysis. Due to the suppression of the quark fluctuations, it is also difficult to explain the $B$ dependence of various gluonic quantities observed in the lattice data [8, 9]. Note also that in the lattice simulations the inverse magnetic catalysis can be observed only when the quark mass is sufficiently small near the physical point [6].

To cure these problems, several authors have emphasized fluctuation effects of various kinds, including hadronic fluctuations $[18,19,20]$ or the back-reaction from quark to gluon sector [21, 22, 23, 24, 25]. Several elaborated model studies took into account these fluctuations, and it became clear that models predicting $M \gg \Lambda_{\mathrm{QCD}}$ do not reproduce the inverse magnetic catalysis $[26,27,28]$. After all, the large fluctuation effects, that is expected to be necessary for the inverse magnetic catalysis phenomenon, are not well activated with such a large mass gap. Note also that the fluctuation arguments alone do not explain why the chiral condensate depends on $B$ linearly.

Keeping these problems in mind, we proposed that the quark mass gap can stay around $\sim \Lambda_{\mathrm{QCD}}$ even for $|e B| \gg \Lambda_{\mathrm{QCD}}^{2}[12]$. If this is true, we have $\langle\bar{\psi} \psi\rangle_{2 \mathrm{D}} \sim \Lambda_{\mathrm{QCD}}$ so that $\langle\bar{\psi} \psi\rangle_{4 \mathrm{D}} \sim|e B| \Lambda_{\mathrm{QCD}}$. In addition, at larger $B$ quark fluctuations are no longer suppressed, but enhanced due to the IR phase space extended by the magnetic fields. Indeed, a perturbative effective potential at a fixed effective quark mass indicates the reduction of $T_{c}$ as $B$ increases [29]. All these features seem consistent with the lattice results.

Of course, the question is how to get the mass gap of $\sim \Lambda_{\mathrm{QCD}}$. The estimate strongly depends 


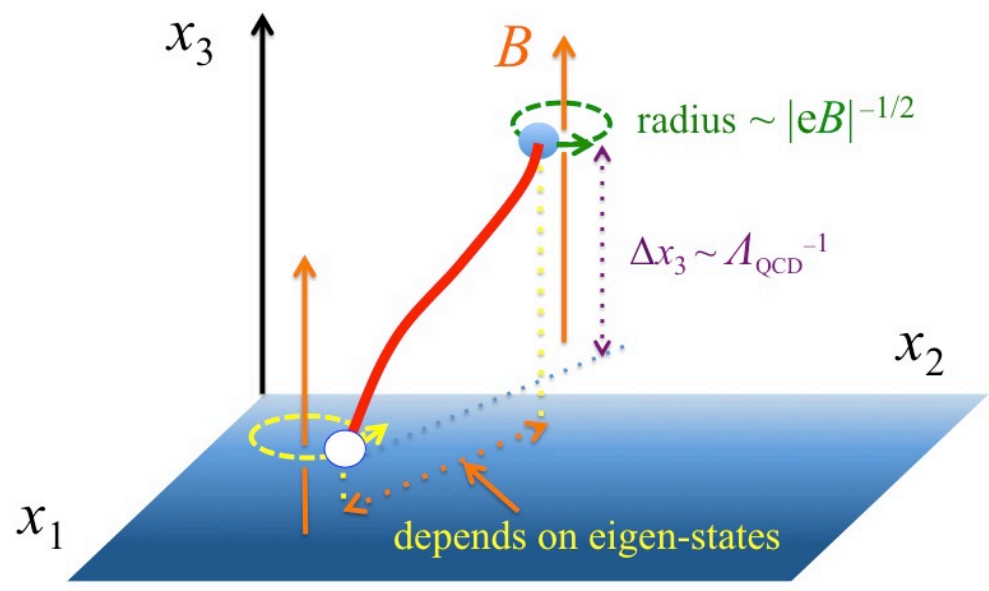

Figure 1: A schematic picture for a meson state to be discussed in this paper. A quark and an anti-quark wrap around magnetic fluxes and the radius of the trajectories is $\sim|B|^{-1 / 2}$. The distance between these two trajectories is $\sim \Lambda_{\mathrm{QCD}}^{-1}$ in the direction of magnetic fields, while the transverse distance depends on the eigen-states; in low energy bound states the transverse distance are much smaller than $\Lambda_{\mathrm{QCD}}^{-1}$, saving the length of the color-flux-tube.

on the nature of the interactions. As argued in the previous works [12], the $B$-dependence drops off from the mass gap if the IR contributions of the interactions dominate over the UV contributions. This observation is consistent with the Schwinger-Dyson studies in which the IR enhanced force was examined $[12,30]$. This feature is presumably very specific to non-Abelian gauge theories, not present in typical effective models.

One of the weakness in our arguments was that the quark self-energies are generally gaugedependent quantities, and sometimes our intuition does not work well for such objects. For example, the quark self-energies can be IR divergent in Coulomb gauge type confining models [31]. These artifacts disappear at the level of color-singlet objects for which artificial contributions cancel each other, leaving only physical quantities. Thus in this paper we will try to deduce (or define) the constituent quark mass out of the hadron spectra; in fact this is how the concept of the constituent quark mass has been developed in traditional arguments.

This observation motivates us to study mesons in strong magnetic fields ${ }^{2}$. In the present context, a sketchy argument was already presented in Ref.[12], while in this paper we will give a more elaborate account of meson properties. We focus on mesons of light flavors with quantum numbers allowing a quark and an anti-quark to stay at the LLL. Other mesons that inevitably

\footnotetext{
${ }^{2}$ In this paper the QED part is essentially quenched, and the magnetic field is always uniform. The possibility of non-uniform distributions of vortices due to the $\rho$-meson condensates (see e.g. Refs.[32]) is omitted from the very beginning by our setup.
} 
contain higher LLs at any time slice have the energy of $\sim \sqrt{|e B|}$ and will not be discussed. In this selection, neutral mesons as well as charged mesons come into our game, and they are as light as $\sim \Lambda_{\mathrm{QCD}}$. Studies of neutral mesons require treatments beyond those by purely hadronic models, since at large $B$ magnetic fluxes penetrate hadrons and couple to quarks inside. This causes the structural change in neutral mesons $[12,18,33,34,35,36]$. For the lattice studies, see Refs.[37, 38]. As an example, in Fig.1 we show a schematic picture of a meson state to be discussed in this paper (see Sec.4 for the outline).

We will study mesons at finite transverse momenta for construction of the hadron resonance gas (HRG) model at finite $B$. The weak field regime was studied in the purely hadronic description in Ref. [39, 40], while our concern in this paper is the strong field regime which requires considerations at the quark level. Our approach has some overlap in philosophy with those in Refs. [18, 41], although the details are different. We will find that the transverse momentum dependence in the meson spectrum is sensitive to the nature of the interactions. In particular, if the IR component dominates, the energy cost associated with the transverse momenta is suppressed by $\sim B^{-2}$ for neutral mesons and $\sim|B|^{-1}$ for charged mesons, and thereby the meson energy grows very slowly as a function of the transverse momentum. This allows many meson states to stay at low energy. At finite temperature, the HRG pressure grows with an increasing $B$, approaching the percolation or chiral restoration regimes at lower $T$ compared to the $B=0$ case. This would offer a gauge invariant description for the inverse magnetic catalysis.

For all these purposes, we analyze the structures of the Schwinger-Dyson and Bethe-Salpeter equations. Most of parametric estimates will be given assuming the long-range interactions, such as linear rising or harmonic oscillator potentials. The use of them provides illuminating predictions dramatically different from models of short-range interactions. We will address why such difference emerges.

Our analyses for the quark self-energy and bound state spectra are essentially based on the large $N_{\mathrm{c}}$ approximation in which the back reaction from the quark sector to the gluon sector can be ignored. For example, the magnetic field induced quark screening effects for long-range forces, such as the reduction of the string tension observed in the lattice QCD [9], will not be manifestly taken into account. But those effects enhance the inverse magnetic catalysis behavior and are in line with our original proposal. In this paper we will show that even without these back reaction effects one can address a plausible mechanism to explain the inverse magnetic catalysis.

This paper is intended for the first part of a series of papers. In this paper we try to address generic aspects of meson problems, leaving more quantitative and model-dependent estimates for the second paper.

This paper is structured as follows. In Sec. 2, we introduce the Ritus bases for fermions, and discuss the $B$-dependent form factor and Schwinger phase which arise from the quark-gluon 
vertices. We argue why the LLL and higher LLs tend to decouple at large $B$ in asymptotic free theories. In Sec. 3, we analyze general structures of the Schwinger-Dyson equation. In Sec. 4 we proceed to analyses on the structure of the Bethe-Salpeter equations for neutral and charged mesons. More specific aspects are elaborated in Secs. 5 and 6 for neutral and charged mesons, respectively. In Sec.7, we discuss the meson resonance gas, using the knowledges deduced in the previous sections. Sec. 8 is devoted to summary and outlook. In Appendix A, we discuss some operator relations which are useful to classify the meson states made of quarks in the LLL.

\section{QCD interactions in magnetic fields}

\subsection{Decomposition by Ritus bases}

In the presence of a magnetic field $B$, our tree level Langrangian reads

$$
\mathcal{L}_{0}=\sum_{f} \bar{\psi}_{f}\left(\not \mathcal{D}+m_{f}\right) \psi_{f}, \quad \mathcal{D}_{\mu}=\partial_{\mu}+\mathrm{i} e_{f} \mathcal{A}_{\mu}
$$

where $\psi_{f}$ is a quark field with the flavor index $f$ and the current quark mass $m_{f}$, and $\mathcal{A}_{\mu}$ is a $U_{\text {em }}(1)$ external gauge field. We use the Euclidean signature $g_{\mu \nu}=\delta_{\mu \nu}$ so that we do not have to distinguish $x_{\mu}$ and $x^{\mu}$. The $\gamma$-matrices are defined as Hermitian matrices, $\gamma_{\mu}^{\dagger}=\gamma_{\mu}$ and $\gamma_{5}=\gamma_{0} \gamma_{1} \gamma_{2} \gamma_{3}=\gamma_{5}^{\dagger}$. Below we use notations, $x_{L}=\left(x_{0}, x_{3}\right), x_{\perp}=\left(x_{1}, x_{2}\right)$, and the counterparts for momenta, assuming that a uniform magnetic field is applied in the $x_{3}$-direction. We choose the Landau gauge $\mathcal{A}_{\perp}=\left(0, B x_{1}\right)$, and will find that this choice simplifies an issue of gauge dependence in the Schwinger-Dyson equation. For later convenience, we also introduce the shorthand notations, $B_{f} \equiv e_{f} B$ and $s_{f} \equiv \operatorname{sgn}\left(B_{f}\right)$, where sgn is the sign function.

There are three steps to construct the Ritus bases. (i) The first step is to project out the spin of charged fermions as the energy levels in a magnetic field are subject to the Zeeman effect. This can be done by using the spin-projection operators $\mathcal{P}_{ \pm}^{f}=\left(1 \pm s_{f} \mathrm{i} \gamma_{1} \gamma_{2}\right) / 2$ as

$$
\psi_{ \pm}^{f} \equiv \mathcal{P}_{ \pm}^{f} \psi^{f}, \quad \mathrm{i} \gamma_{1} \gamma_{2} \psi_{ \pm}^{f}= \pm s_{f} \psi_{ \pm}^{f}
$$

It is important to remember that $\mathcal{P}_{ \pm}$commutes with $\gamma_{L}$, while anti-commute with $\gamma_{\perp}$ which flips the spin. (ii) Secondly, noting that momenta $p_{L}$ and $p_{2}$ are conserved in the Landau gauge, we can expand fermion fields as

$$
\psi_{ \pm}^{f}(x)=\sum_{l=0}^{\infty} \int \frac{\mathrm{d}^{2} p_{L} \mathrm{~d} p_{2}}{(2 \pi)^{3}} \psi_{ \pm}^{f}\left(l, p_{2}, p_{L}\right) \mathcal{H}_{l}^{f}\left(x_{1}-r_{p_{2}}^{f}\right) \mathrm{e}^{\mathrm{i} p_{2} x_{2}} \mathrm{e}^{\mathrm{i} p_{L} x_{L}}, \quad r_{p_{2}}^{f} \equiv-\frac{p_{2}}{B_{f}}
$$

where the index $l$ labels an orbital level in the transverse motion. $\mathcal{H}_{l}^{f}$ is the normalized harmonic oscillator function. In this work, we will use the explicit form at $l=0$ which is given by

$$
\mathcal{H}_{0}^{f}\left(x_{1}-r_{p_{2}}^{f}\right)=\left(\frac{\left|B_{f}\right|}{\pi}\right)^{1 / 4} \exp \left[-\frac{\left|B_{f}\right|}{2}\left(x_{1}-r_{p_{2}}^{f}\right)^{2}\right] .
$$


Note that the principal quantum number $n$ appearing in the energy level (1) is the sum of the orbital level index $l$ and the Zeeman splitting $\pm g / 2$ with the $g$-factor $(g=2)$, i.e., $n=2 l+1 \pm 1$; the energy levels are two-fold degenerated except for the unique ground state $n=0$. (iii) Therefore, the last step is to relabel the fermion fields since the orbital level indices are not conserved numbers even at the tree level. We set

$$
\chi_{p_{2}}^{f}\left(p_{L}\right) \equiv \psi_{n=0, p_{2}}^{f}\left(p_{L}\right) \equiv \psi_{+}^{f}\left(l=0, p_{2}, p_{L}\right)
$$

for the LLL, and

$$
\psi_{n, p_{2}}^{f+}\left(p_{L}\right) \equiv \psi_{+}^{f}\left(l=n, p_{2}, p_{L}\right), \quad \psi_{n, p_{2}}^{f-}\left(p_{L}\right) \equiv \psi_{-}^{f}\left(l=n-1, p_{2}, p_{L}\right),
$$

for the higher LLs (hLLs). We also define $\psi_{n, p_{2}}^{f} \equiv \psi_{n, p_{2}}^{f+}+\psi_{n, p_{2}}^{f-}$, which will be useful to have a concise expression of the action below.

Then the zero-th order quark action for a flavor $f$ can be written as a sum of the Landau levels as $\mathcal{L}^{f}=\mathcal{L}_{\chi}^{f}+\sum_{n=1} \mathcal{L}_{n}^{f}$, where

$$
\int_{x} \mathcal{L}_{\chi}^{f}=\int_{p_{L}, p_{2}} \bar{\chi}_{p_{2}}^{f}\left(p_{L}\right)\left(\mathrm{i} p_{L}+m_{f}\right) \mathcal{P}_{+}^{f} \chi_{p_{2}}^{f}\left(p_{L}\right)
$$

for the LLL, and

$$
\sum_{n=1} \int_{x} \mathcal{L}_{n}^{f}=\sum_{n=1} \int_{p_{L}, p_{2}} \bar{\psi}_{n, p_{2}}^{f}\left(p_{L}\right)\left(\mathrm{i} \not \not_{n}^{f}+m_{f}\right) \psi_{n, p_{2}}^{f}\left(p_{L}\right)
$$

for the hLLs with $\left(P_{n}^{f}\right)_{\perp} \equiv\left(0, s_{f} \sqrt{2 n\left|B_{f}\right|}\right)$. Note that the $B$-dependence drops off from the tree-level Lagrangian for the LLL, as emphasized in Introduction.

\subsection{Quark-gluon vertices with form factors and Schwinger phases}

While the Ritus bases diagonalize the tree-level Lagrangian, interactions induces transitions between different LLs at the quark-gluon vertices. In particular the $B$-dependence of the LLL appears only through the interactions. Using the expansion (5) by the Ritus basis, the vertex of a $f$-flavored quark reads

$$
\begin{aligned}
\mathrm{i} g_{s} \int_{x} \bar{\psi}_{f} \gamma_{\mu} t_{a} \psi_{f} A_{\mu}^{a}(x)=\mathrm{i} \sum_{l, l^{\prime}=0}^{\infty} \int_{p_{L}, p_{2}, k_{L}, k_{2}} \bar{\psi}_{f}\left(l, p_{2}, p_{L}\right) \gamma_{\mu} t_{a} \psi_{f}\left(l^{\prime}, k_{2}, k_{L}\right) \\
\quad \times \int_{k_{1}} g_{s} A_{\mu}^{a}(p-k) \int_{x_{1}} \mathcal{H}_{l}^{f}\left(x_{1}-r_{p_{2}}^{f}\right) \mathcal{H}_{l^{\prime}}^{f}\left(x_{1}-r_{k_{2}}^{f}\right) \mathrm{e}^{\mathrm{i}\left(p_{1}-k_{1}\right) x_{1}},
\end{aligned}
$$

where $t_{a}$ is color matrices normalized as $\operatorname{tr}\left[t_{a} t_{b}\right]=\delta_{a b} / 2$, and $A_{\mu}^{a}$ is a gluon field accompanying the coupling constant $g_{s}$. 
We find in Eq. (11) that the integral is factorized because the Ritus bases do not manifestly depend on $k_{1}$ and $x_{1}$. The last integral factor works as an overlap function among the plane-wave basis for a gluon and the Ritus bases for two quarks. Performing the integral, this factor can be written in a product form

$$
\int_{x_{1}} \mathcal{H}_{l}^{f}\left(x_{1}-r_{p_{2}}^{f}\right) \mathcal{H}_{l^{\prime}}\left(x_{1}-r_{k_{2}}^{f}\right) \mathrm{e}^{\mathrm{i}\left(p_{1}-k_{1}\right) x_{1}} \equiv \mathrm{e}^{\mathrm{i} \Xi_{p, k}^{f}} \mathcal{R}_{l l^{\prime}}^{f}\left(\vec{p}_{\perp}-\vec{k}_{\perp}\right) .
$$

The phase factor $\Xi_{p, k}^{f}$ is called the Schwinger phase, and its explicit form is given by

$$
\Xi_{p, k}^{f} \equiv\left(p_{1}-k_{1}\right) r_{\left(p_{2}+k_{2}\right) / 2}^{f}=-\left(p_{1}-k_{1}\right) \frac{p_{2}+k_{2}}{2 B_{f}},
$$

which depends upon $\left(p_{2}+k_{2}\right) / 2$, that is, the average of fermion momenta before and after the interaction. This exponent is gauge variant; the residual symmetry in the Landau gauge, $\mathcal{A}_{2}=$ $B x_{1} \rightarrow B\left(x_{1}+c\right)$ preserves magnetic fields but shifts the fermion momenta in the $x_{2}$-direction. An advantage in the Landau gauge is that the exponent is common to all of the combinations of couplings between different $\mathrm{LLs}^{3}$. We will see that the Schwinger phase provides physical effects when we calculate many-body states.

The other factor at the vertex depends only on the momentum transfer flowing into the gluon line as

$$
\mathcal{R}_{l l^{\prime}}^{f}\left(\vec{p}_{\perp}-\vec{k}_{\perp}\right) \equiv \int_{x_{1}} \mathcal{H}_{l}^{f}\left(x_{1}-r_{\left(p_{2}-k_{2}\right) / 2}^{f}\right) \mathcal{H}_{l^{\prime}}^{f}\left(x_{1}+r_{\left(p_{2}-k_{2}\right) / 2}^{f}\right) \mathrm{e}^{\mathrm{i}\left(p_{1}-k_{1}\right) x_{1}}
$$

We call it the form factor. For the momentum transfer $\vec{q}_{\perp}=\vec{p}_{\perp}-\vec{k}_{\perp}$, we find

$$
\mathcal{R}_{l l^{\prime}}\left(\vec{q}_{\perp}\right) \propto\left\{\begin{array}{ll}
\left(\frac{q_{1}-\mathrm{i} s_{f} q_{2}}{\left|2 B_{f}\right|^{1 / 2}}\right)^{l-l^{\prime}} & \left(l>l^{\prime}\right) \\
\left(\frac{q_{1}+\mathrm{i} s_{f} q_{2}}{\left|2 B_{f}\right|^{1 / 2}}\right)^{l^{\prime}-l} & \left(l^{\prime}>l\right)
\end{array} .\right.
$$

When $l \neq l^{\prime}$, these power factors weaken the relevance of interactions in the IR regime, $q_{1,2} \lesssim \sqrt{B_{f}}$. Thus the $l \neq l^{\prime}$ processes, in which a quark hops from $l$-th to $l^{\prime}$-th orbital level, are dominated by the UV interactions. In asymptotic free theories, these UV interactions are in the weakcoupling regime and can be treated within the perturbation theory. On the other hand, such IR suppressions do not occur for the $l=l^{\prime}$ processes, so that results are sensitive to the IR structure of the interactions. Therefore, one must directly handle the non-perturbative features of gluon propagators and quark-gluon vertices explicitly.

3 This simplification is by no means trivial. In fact, in the symmetric gauge, it is difficult to disentangle the Schwinger's phase part and the others; the expansion of the Schwinger's phase appears as powers of the pseudo angular momenta, and apparently the fermions labeled by different pseudo angular momenta acquire different self-energies. The Schwinger's phase can be factored out only after summing up all powers of the pseudo angular momenta that yields the exponentiated form. 


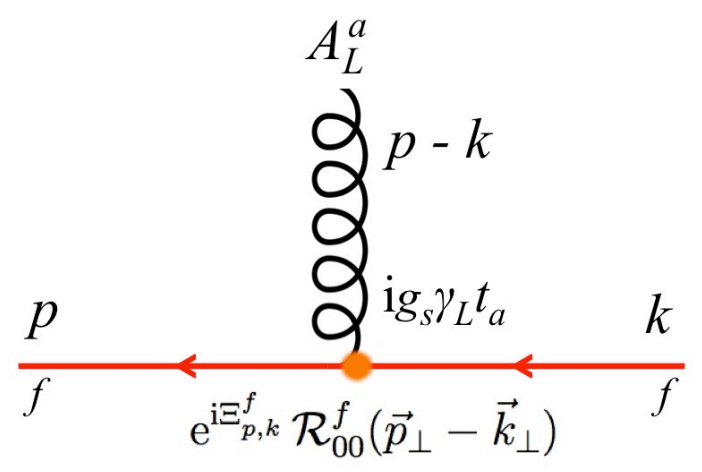

Figure 2: The quark-gluon vertex for the LLL with the flavor $f$.

\subsection{Interactions for the $L L L$}

In this work we focus on the LLL in the strong field limit. As depicted in Fig. 2, the interaction Lagrangian is given by

$$
\mathcal{L}_{\mathrm{LLL}}^{\mathrm{int}}=\mathrm{i} \sum_{f} \int_{p_{L}, p_{2}, k_{L}, k_{2}} \bar{\chi}_{p_{2}}^{f}\left(p_{L}\right) \gamma_{L} t_{a} \mathcal{P}_{+}^{f} \chi_{k_{2}}^{f}\left(k_{L}\right) \int_{k_{1}} \mathrm{e}^{\mathrm{i} \Xi_{p, k}^{f}} \mathcal{R}_{00}^{f}\left(\vec{p}_{\perp}-\vec{k}_{\perp}\right) \times g_{s} A_{L}^{a}(p-k) .
$$

Note that the $\gamma_{\perp}$ component drops off because $\mathcal{P}_{+} \gamma_{\perp} \mathcal{P}_{+}=0$. More physically, the $\gamma_{\perp}$ vertices flip spins and inevitably couple the LLL to hLLs, so that it is irrelevant when we consider only the low energy sector. This observation also suggests that the relevant gauge fields at the low energy are $A_{L}$, while $A_{\perp}$ 's tend to decouple from the low energy dynamics. The lattice simulations for gluon condensates and string tensions have indeed shown that configurations of $A_{L}$ are more screened than $A_{\perp}$, and this behavior can be understood from the suppression of the coupling to $A_{\perp}$ at the LLL [8, 9].

A final comment is on the form factor. One finds its explicit form at the LLL as

$$
R_{00}^{f}\left(\vec{q}_{\perp}\right)=\mathrm{e}^{-\frac{\vec{q}_{\perp}^{2}}{4\left|B_{f}\right|}}
$$

which cuts off the UV contribution when $\vec{q}_{\perp}^{2}$ is larger than $\left|B_{f}\right|$. In other words, the size of $\left|B_{f}\right|$ determines to what extent the UV sector can participate in the LLL dynamics. There is another important remark: at $\vec{q}_{\perp}^{2} \ll\left|B_{f}\right|$, the form factor behaves as

$$
R_{00}^{f}\left(\vec{q}_{\perp}\right) \simeq 1-\frac{\vec{q}_{\perp}^{2}}{4\left|B_{f}\right|}+\cdots, \quad\left(\vec{q}_{\perp}^{2} \ll\left|B_{f}\right|\right)
$$

which means that the $B$-dependence can be dropped off from the IR sector.

With these observations, now we can see that there are two distinct contributions in the interactions, namely, the magnetic-field dependent contributions from the UV interactions, and magnetic-field independent contributions from the IR interactions. The $B$-dependence of the quark 


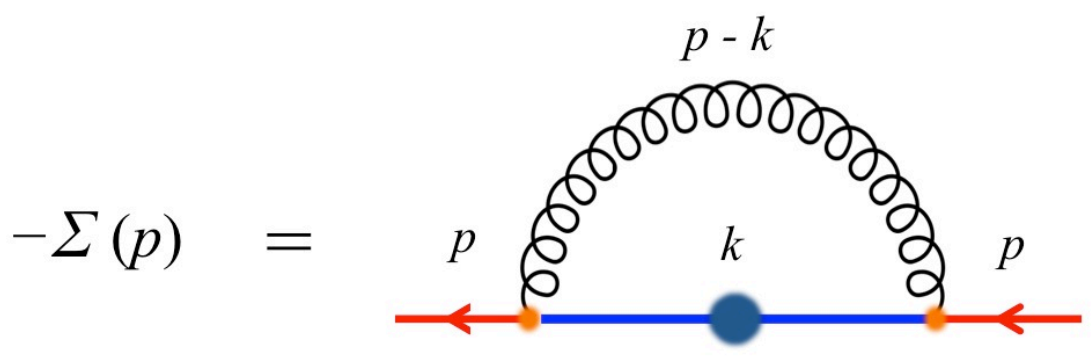

Figure 3: The Schwinger-Dyson equation within the rainbow approximation.

self-energies, chiral condensates, meson spectra, and so on, is determined by the competition between the UV and IR contributions, and thus is very sensitive to the fundamental nature of theories one considers. For instance, if one employs the models involving contact interactions, the UV contributions are strongly enhanced, and then the quark self-energies are very sensitive to the value of $B$. In contrast, theories involving stronger IR interactions show a much weaker $B$-dependence. Therefore, in this respect, QCD should be distinguished from the models that do not properly include the dynamics of the gluonic sector.

\section{Schwinger-Dyson equations for the LLL: General analyses}

This section is devoted to general analyses on the Schwinger-Dyson equations for the selfenergy of the LLL. For simplicity we consider the rainbow approximation only, but we expect that our arguments hold even after this restriction is removed. Assuming the strong field limit, we restrict both the external and intermediate quark states to the LLL. For $|e B|>0.3 \mathrm{GeV}^{2}$, the impact of hLL intermediate states to the LLL self-energies was found to be small $^{4}$, so we may ignore it [42].

The dressed quark propagator for the LLL can be written as

$$
\left.S\left(q_{L}, \boldsymbol{\Sigma}(q)\right)=[\mathrm{i}(\not q+\not))_{L}+\left(m+\Sigma_{m}\right)\right]^{-1} \mathcal{P}_{+}=S_{0}+S_{0}(-\Sigma) S_{0}+\cdots
$$

where $S_{0}(q)=\left(\mathrm{i} \phi_{L}+m\right)^{-1}$ is the tree propagator of the LLL, and for the moment we suppress

4 The large energy gap between the LLL and the hLL itself does not justify the LLL approximation, because an infinite sum of small contributions of the order of $|B|^{-1}$ produces a UV divergence. However, this UV divergence should be nothing but the divergence in the vacuum at $B=0$. Therefore, the divergence can be absorbed into the renormalized current quark mass at $B=0$, and the remaining hLL contributions result in only (very) small higher order $B$-corrections [42]. In short, the summation of discretized energy levels and the integral of continuum states yield similar results, so details of the hLL contributions do not play a major role in the LLL self-energies. 
flavor indices. The self-energy $\Sigma$ is decomposed into

$$
\Sigma \equiv \mathrm{i} \mathbb{Z}_{L}+\Sigma_{m}
$$

We have dropped off the $\Sigma_{\perp}$ component because the $\gamma_{\perp}$ structure is forbidden by the projection operators as $\mathcal{P}_{+} \gamma_{\perp} \mathcal{P}_{+}=0$. Denoting the gluon propagator as $D_{\mu \nu}^{a b}=\delta^{a b} D_{\mu \nu}$, the Schwinger-Dyson equation is then given by

$$
-\Sigma(p)=(-\mathrm{i})^{2} C_{F} \int_{k_{L}, \vec{k}_{\perp}} \gamma_{L^{\prime}} S\left(k_{L} ; \Sigma(k)\right) \gamma_{L^{\prime \prime}} \times D_{L^{\prime} L^{\prime \prime}}(p-k)\left[\mathcal{R}_{00}\left(\vec{p}_{\perp}-\vec{k}_{\perp}\right)\right]^{2}
$$

where $C_{F}=\left(N_{\mathrm{c}}^{2}-1\right) / 2 N_{\mathrm{c}}$ is the Casimir for the fundamental representation. We will keep the general form of the gluon propagator $D_{\mu \nu}$ which includes insertions of gluon self-energies and is convoluted with dressed vertices. We emphasize that in the self-energy calculations the Schwinger phases from two vertices exactly cancel out.

Next, we will show that the self-energy is independent of the transverse momenta even after radiative corrections are included. To prove it, we compare the self-energies $\Sigma\left(p_{L}, \vec{p}_{\perp}\right)$ and $\Sigma\left(p_{L}, \vec{p}_{\perp}+\delta \vec{p}_{\perp}\right)$. First we notice that, in contrast to the $B=0$ cases, the propagator depends on $p_{\perp}$ only through the self-energy part. Secondly, the integrand other than the propagator part depends only on the difference $\vec{p}-\vec{k}$. Thus, by shifting the integration variable $\vec{k}_{\perp}$, we find

$$
\begin{aligned}
& \boldsymbol{\Sigma}\left(p_{L}, \vec{p}_{\perp}+\delta \vec{p}_{\perp}\right)-\Sigma\left(p_{L}, \vec{p}_{\perp}\right) \\
& \propto \int_{k_{L}, \vec{k}_{\perp}}\left[S\left[k_{L} ; \boldsymbol{\Sigma}\left(k_{L}, \vec{k}_{\perp}+\delta \vec{p}_{\perp}\right)\right]-S\left[k_{L} ; \boldsymbol{\Sigma}\left(k_{L}, \vec{k}_{\perp}\right)\right]\right] \times D_{L^{\prime} L^{\prime \prime}}(p-k)\left[\mathcal{R}_{00}\left(\vec{p}_{\perp}-\vec{k}_{\perp}\right)\right]^{2} .
\end{aligned}
$$

This relation must hold for any values of $\delta \vec{p}_{\perp}$. An obvious candidate is $\Sigma\left(p_{L}, \vec{p}_{\perp}\right)=\Sigma\left(p_{L}\right)$, which is independent of $\vec{p}_{\perp}$; in this case the LHS and RHS both vanish in the above equation. This is a natural solution as the perturbative calculation also results in the $\vec{p}_{\perp}$-independent self-energy.

With the above solution, we may write $\Sigma(q)=\Sigma\left(q_{L}\right)$, and then find that the integral equation (21) is factorized as follows. The separation between the longitudinal and transverse momentum dependences allows us to define a two-dimensional effective propagator by

$$
D_{L^{\prime} L^{\prime \prime}}^{2 \mathrm{D}}\left(p_{L}-k_{L}\right) \equiv C_{F} \int_{\vec{k}_{\perp}} D_{L^{\prime} L^{\prime \prime}}(p-k)\left[\mathcal{R}_{00}\left(\vec{p}_{\perp}-\vec{k}_{\perp}\right)\right]^{2}
$$

and the dimensionally reduced Schwinger-Dyson equation in the $(1+1)$ dimensions by

$$
\mathrm{i} \oiint_{L}\left(p_{L}\right)+\Sigma_{m}\left(p_{L}\right)=\int_{k_{L}} \gamma_{L^{\prime}} S\left(k_{L}\right) \gamma_{L^{\prime \prime}} \times D_{L^{\prime} L^{\prime \prime}}^{2 \mathrm{D}}\left(p_{L}-k_{L}\right)
$$

(We should remember that the flavor indices for $\Sigma, S$, and $D^{2 \mathrm{D}}$ are suppressed.)

In this equation, the only one origin of the $B$-dependence is the form factor hidden in the definition of $D^{2 \mathrm{D}}$, since the LLL propagator does not contain any manifest $B$-dependence. Therefore 

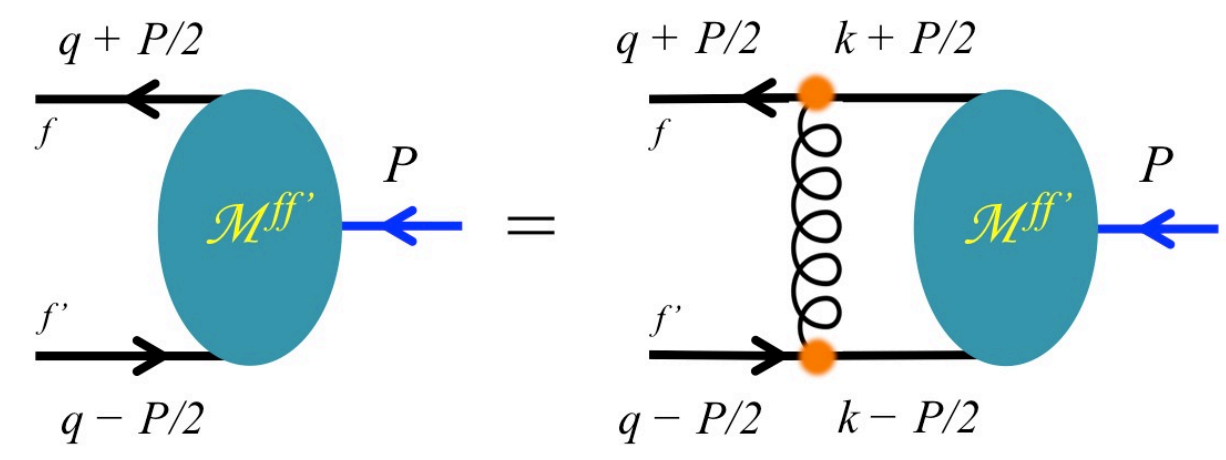

Figure 4: The (homogeneous) Bethe-Salpeter equation within the rainbow ladder approximation.

our problem to examine the $B$-dependence is now reduced to the study of the effective propagator; if the effective propagator strongly depends on $B$, the self-energies also do; if not, we will find the self-energies (nearly) independent of $B$, which, as mentioned in Introduction, provides the key ingredient of the scenario for the magnetic catalysis and inverse catalysis of the chiral condensate observed by the lattice QCD simulations.

\section{The Bethe-Salpeter equations for the LLL: General analyses}

\subsection{Some definitions}

In this section we investigate general structures of the Bethe-Salpeter equations for bound states composed of the fermions in the LLL, and discuss characteristic differences between neutral and charged mesons. The self-energies discussed in the previous section is supposed to be used as an input to the Bethe-Salpeter equation. We consider the Bethe-Salpeter amplitude defined by

$$
\left\langle 0\left|\bar{\chi}_{q_{2}^{-}}^{f^{\prime}}\left(q_{L}^{-}\right) \chi_{q_{2}^{+}}^{f}\left(q_{L}^{+}\right)\right| \mathcal{M}_{P_{2}^{\prime} P_{L}^{\prime}}\right\rangle \equiv(2 \pi)^{3} \delta\left(P_{2}-P_{2}^{\prime}\right) \delta\left(P_{3}-P_{3}^{\prime}\right) \delta\left(\mathrm{i} P_{0}+E\left(P_{3}^{\prime}\right)\right) \times \mathcal{M}_{P_{2} P_{3}}^{f f^{\prime}}\left(q_{2}, q_{L}\right),
$$

where $\left(P_{2}, P_{L}\right)$ are conserved total momenta, and $q_{L}^{ \pm}=q_{L} \pm P_{L} / 2$ and $q_{2}^{ \pm}=q_{2} \pm P_{2} / 2$ are momenta carried by a quark and an anti-quark, respectively (see Fig.4). Especially we will be interested in the equal-time amplitude, which is defined as

$$
\mathcal{M}_{P_{2} P_{3}}^{f f^{\prime}}\left(q_{2}, q_{3}\right) \equiv \int_{q_{0}} \mathcal{M}_{P_{2} P_{3}}^{f f^{\prime}}\left(q_{2}, q_{L}\right)
$$

in which the $\chi^{f^{\prime}}$ and $\chi^{f}$ hit the meson state at the same time. Using relative and center-of-mass (COM) coordinates, $r_{i}=x_{i}-x_{i}^{\prime}$ and $R_{i}=\left(x_{i}+x_{i}^{\prime}\right) / 2$, respectively, the coordinate-space expression can be constructed as

$$
\mathcal{M}_{P_{2} P_{3}}^{f f^{\prime}}(\vec{r} ; \vec{R}) \equiv \mathcal{N} \mathrm{e}^{\mathrm{i}\left(P_{2} R_{2}+P_{3} R_{3}\right)} \int_{q_{2}, q_{3}} \mathrm{e}^{\mathrm{i}\left(q_{2} r_{2}+q_{3} r_{3}\right)} \mathcal{H}_{0}^{f}\left(x_{1}-r_{q_{2}^{+}}^{f}\right) \mathcal{H}_{0}^{f^{\prime}}\left(x_{1}^{\prime}-r_{q_{2}^{-}}^{f^{\prime}}\right) \mathcal{M}_{P_{2} P_{3}}^{f f^{\prime}}\left(q_{2}, q_{3}\right)
$$


where $\mathcal{N}$ is some normalization factor. (Here we included $\mathrm{e}^{\mathrm{i} P_{2} R_{2}}$ in the definition since the integral still contains $P_{2}$ which will be combined with the exponential factor.)

From this form of the wavefunction, we can already get some insights on the bound state problem. At a large $B$, the probability distributions in the $x_{1}$-direction are localized around $r_{q_{+}}^{f}$ and $r_{q_{-}}^{f^{\prime}}$ with the variances of $\sim 1 /|B|^{1 / 2}$, reflecting the cyclotron motion of each quark. Therefore the relative and COM coordinates are roughly given by combinations of the conserved momentum $P_{2}$ and the expectation value of the relative momentum $\left\langle q_{2}\right\rangle$ as

$$
\begin{aligned}
& \left\langle R_{1}\right\rangle \sim\left(\frac{1}{B_{f}}+\frac{1}{B_{f^{\prime}}}\right)\left\langle q_{2}\right\rangle+\left(\frac{1}{B_{f}}-\frac{1}{B_{f^{\prime}}}\right) \frac{P_{2}}{2}, \\
& \left\langle r_{1}\right\rangle \sim\left(\frac{1}{B_{f}}-\frac{1}{B_{f^{\prime}}}\right)\left\langle q_{2}\right\rangle+\left(\frac{1}{B_{f}}+\frac{1}{B_{f^{\prime}}}\right) \frac{P_{2}}{2} .
\end{aligned}
$$

To get intuitive pictures for our bound state problem, first we stress that the momenta we are dealing with are the "canonical" momenta which are gauge-variant. After making a gauge choice, this momentum is related to the "guiding center" around which particles exhibit the cyclotron motion. More intuitively appealing quantity is the "kinetic" momentum, $p+e_{f} A$, which is related to the velocity. For instance a particle at large $x_{1}$ has large canonical momentum $k_{2} \simeq-B_{f} x_{1}$ but also large $e_{f} A_{2}=B_{f} x_{1}$. As the sum of them, the kinetic momentum is not large and remains $\sim\left|B_{f}\right|^{1 / 2}$ as expected from the cyclotron motion; a particle with large $k_{2}$ has neither large velocity nor large Lorentz force.

Except the quantum aspects related to the vector potential and guiding center, the remaining part can be understood within the classical picture. Like the (classical) Hall effect, a particle (anti-particle) drifts in the direction orthogonal to those of magnetic fields and a force acting on it. The direction of the drift depends on the electric charge. Now let us examine two characteristic examples.

In case of $e_{f}=e_{f^{\prime}}$ for the neutral mesons [Fig.5(left)], we have

$$
\left\langle R_{1}\right\rangle_{e_{f}=e_{f^{\prime}}} \sim \frac{2\left\langle q_{2}\right\rangle}{B_{f}}, \quad\left\langle r_{1}\right\rangle_{e_{f}=e_{f^{\prime}}} \sim \frac{P_{2}}{B_{f}} .
$$

In this case, the relative momentum is related to the COM coordinate. We find that the neutral meson spectra are degenerated with respect to this momentum since the spectra are independent of the location of the COM coordinate in a constant magnetic field. On the other hand, the conserved total momentum $P_{2}$ manifestly enters the bound state problem because, as seen in Eq. (30) and Fig.5, it corresponds to the relative distance between a particle and an anti-particle. In the classical picture, the relative distance is naturally kept fixed since the particle and antiparticle drift in the same direction with the same velocity, as a consequence of $e_{f}=e_{f^{\prime}}$. Moreover this also indicates that besides the conserved momentum $P_{2}$, there should be another constant of 

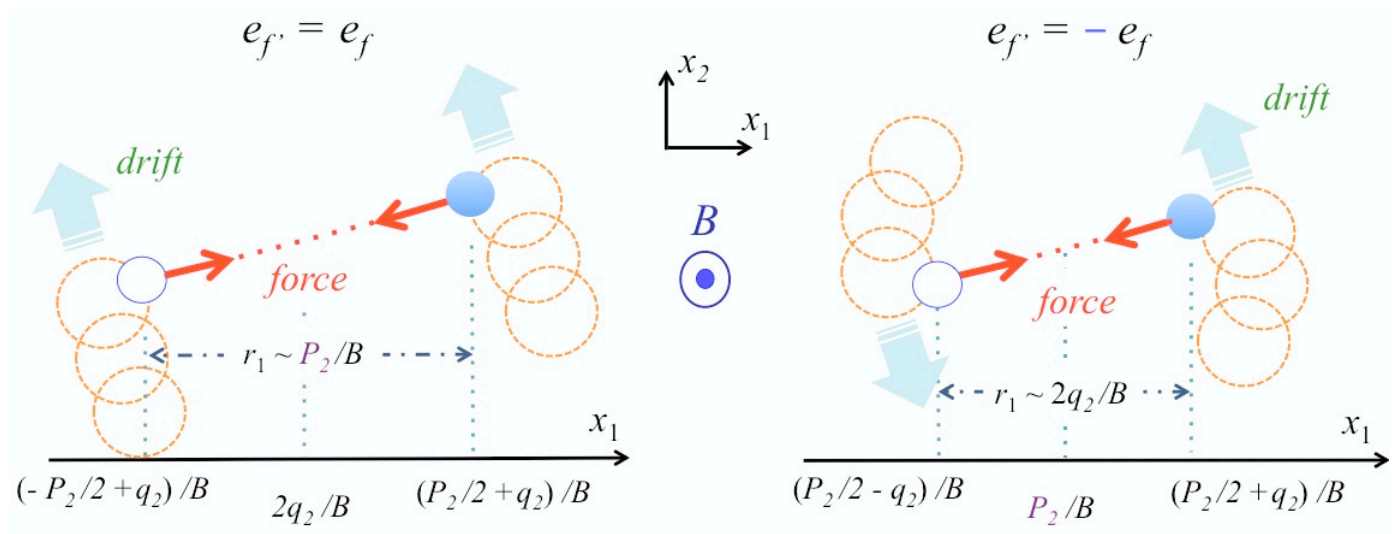

Figure 5: A semi-classical picture for mesons projected onto the plane perpendicular to magnetic fields. (left) The $e_{f^{\prime}}=e_{f}$ case for neutral mesons. A particle and an anti-particle attract each other and drift in the same direction orthogonal to those of the force and magnetic fields (Hall drift). The constants of motion are the relative coordinates with which the strength of the two-body interaction is kept fixed. (right) The $e_{f^{\prime}}=-e_{f}$ case for charged mesons. The directions of the drift are opposite for a particle and an anti-particle, changing the relative coordinate. The constant of motion is the COM in the $x_{1}$-direction $\left(P_{2} / B\right)$, and the typical mode with such conserved quantity is the rotation.

motion that is related to the relative distance in the $x_{2}$-direction. Indeed such conserved quantity appears in our Bethe-Salpeter equation as shown below.

Another characteristic case is bound states of equal charges $e_{f}=-e_{f^{\prime}}$ (Fig.5(right)), which does not exist in the mesonic system (because of the difference between $e_{u}=2 e / 3$ and $e_{d}=-e / 3$ ) but can be applied to diquark or dielectron systems. In such cases, $q_{2}^{-}=q_{2}-P_{2} / 2$ should be replaced by $\tilde{q}_{2}^{-}=-q_{2}+P_{2} / 2$, and this procedure is equivalent to taking $e_{f^{\prime}}=-e_{f}$ in the transverse dynamics of the mesonic systems. Then, from Eqs. (28) and (29), we find

$$
\left\langle R_{1}\right\rangle_{e_{f}=-e_{f^{\prime}}} \sim \frac{P_{2}}{B_{f}}, \quad\left\langle r_{1}\right\rangle_{e_{f}=-e_{f^{\prime}}} \sim \frac{2\left\langle q_{2}\right\rangle}{B_{f}} .
$$

The spectra again should not depend on the location of $R_{1}$, so that the spectra are degenerated with respect to $P_{2}$ in contrast to the case of neutral mesons discussed above. On the other hand, the relative distance $\left\langle r_{1}\right\rangle$ depends on the non-conserved momentum $q_{2}$, leading to nontrivial oscillating modes which couple the motions in the $x_{1^{-}}$and $x_{2}$-directions. In the classical picture, a particle and anti-particle drift in the opposite direction while keeping their COM coordinate in the $x_{1}$-direction at $P_{2} / B_{f}$; this motion around a fixed point can be interpreted as a rotating mode whose radius becomes large for higher excitations.

Below we will provide more precise statements by analyzing the mathematical structure of the Bethe-Salpeter equation, and will see that the above heuristic arguments indeed capture the essence of the bound state problems. 


\subsection{The Bethe-Salpeter equations in the Rainbow Ladder approximation}

In the Rainbow Ladder approximation, the Bethe-Salpeter equation for a pair of fermions in the LLL states is written down as

$$
\begin{aligned}
& \mathcal{M}_{P_{2} P_{3}}^{f f^{\prime}}\left(q_{2}, q_{L}\right)=(-\mathrm{i})^{2} C_{F} \\
& \quad \times S^{f}\left(q_{L}^{+}\right) \gamma_{L}\left[\int_{k_{L}, k_{\perp}} D_{L L^{\prime}}(q-k) \mathcal{F}_{q_{2}^{+}, k_{2}^{+} ; q_{2}^{-}, k_{2}^{-}}^{f f^{\prime}}\left(q_{1}-k_{1}\right) \mathcal{M}_{P_{2} P_{3}}^{f f^{\prime}}\left(k_{2}, k_{L}\right)\right] \gamma_{L^{\prime}} S^{f^{\prime}}\left(q_{L}^{-}\right),
\end{aligned}
$$

where the factor $\mathcal{F}$ summarizes the Schwinger phases (13) and the form factors (17) as

$$
\mathcal{F}_{q_{2}^{+}, k_{2}^{+} ; q_{2}^{-}, k_{2}^{-}}^{f f^{\prime}}\left(q_{1}-k_{1}\right) \equiv \mathcal{R}_{00}^{f} \mathcal{R}_{00}^{f^{\prime}}\left(\vec{q}_{\perp}-\vec{k}_{\perp}\right) \times \mathrm{e}^{\mathrm{i}\left[\Xi_{q_{2}^{+}, k_{2}^{+}}^{f}\left(q_{1}-k_{1}\right)-\Xi_{q_{2}^{-}, k_{2}^{-}}^{f^{\prime}}\left(q_{1}-k_{1}\right)\right]} .
$$

Before examining roles of $\mathcal{F}$, let us first note that the $B$-dependence enters the LLL dynamics only through the factor $\mathcal{F}$. Further we find that the exponents of form factors and Schwinger phases appear with an inverse factor of $1 /|B|$ as $\left(\vec{q}_{\perp}-\vec{k}_{\perp}\right)^{2} /|B|$ and $\left(q_{1}-k_{1}\right) /|B|$, respectively. Therefore, if one plays with theories of the enhanced IR interactions, that factor can be set to $\mathcal{F} \simeq 1$, and then the spectra are of the order of $\Lambda_{\mathrm{QCD}}$ independently of $B$.

The above situation may, however, alter for meson states with large momenta. In such cases,

the Schwinger phases play a dramatically important role. For mesons composed of quarks with $f$ and $f^{\prime}$ flavors, the quantum phase takes the form

$$
\left[\Xi_{q_{2}^{+}, k_{2}^{+}}^{f}-\Xi_{q_{2}^{-}, k_{2}^{-}}^{f^{\prime}}\right]\left(q_{1}-k_{1}\right)=-\left(q_{1}-k_{1}\right) \times\left\{\frac{q_{2}+k_{2}}{2}\left[\frac{1}{B_{f}}-\frac{1}{B_{f^{\prime}}}\right]+\frac{P_{2}}{2}\left[\frac{1}{B_{f}}+\frac{1}{B_{f^{\prime}}}\right]\right\} .
$$

Note that the above expression depends on both the total momentum $P_{2}$ and $\left(q_{2}+k_{2}\right) / 2$ which is the average relative momentum before and after the interaction. Clearly, this factor manifestly couples the COM dynamics to the relative dynamics between two particles, i.e., the COM and relative motions are not independent of each other. In the subsequent sections, we will examine how this factor plays a role in the $e_{f}=e_{f^{\prime}}$ and $e_{f} \neq e_{f^{\prime}}$ cases more specifically.

\section{Neutral mesons : the $e_{f}=e_{f^{\prime}}$ case}

For $e_{f}=e_{f^{\prime}}$, the quantum phase (34) in the last section becomes

$$
\left[\Xi_{q_{2}^{+}, k_{2}^{+}}^{f}-\Xi_{q_{2}^{-}, k_{2}^{-}}^{f}\right]\left(q_{1}-k_{1}\right)=-\left(q_{1}-k_{1}\right) \frac{P_{2}}{B_{f}}
$$

where the dependence on the average relative momentum $\left(q_{2}+k_{2}\right) / 2$ drops off, while the total momentum $P_{2}$ remains. There is another special character in the $e_{f}=e_{f^{\prime}}$ case. As shown below, we can define a (fictious) transverse momentum $P_{1}$, in addition to the conversed momentum $P_{2}$ which already exists in Eq. (35). 
By making use of the additional momentum $P_{1}$, we can transform the Bethe-Salpeter equation into a rotationally symmetric form. To see this, we shall first determine the $q_{2}$-dependence of the amplitude. To simplify expressions, we suppress irrelevant indices and arguments such as $q_{L}, k_{1}, \cdots$, and write the structure of the Bethe-Salpeter equation in the following symbolic form

$$
\mathcal{M}\left(q_{2}\right)=\int_{k_{2}} \mathcal{K}\left(q_{2}-k_{2}\right) \mathcal{M}\left(k_{2}\right)
$$

The kernel $\mathcal{K}$ depends on $q_{2}$ and $k_{2}$ only through the difference $q_{2}-k_{2}$. This is because (i) the Schwinger phase does not depend on $q_{2}+k_{2}$ specifically for the $e_{f}=e_{f^{\prime}}$ case, and (ii) the fermion propagators are independent of $k_{2}$ and $q_{2}$. The latter is a special character of the Landau level propagators. With this symbolic form at hand, one can conclude that if $\mathcal{M}\left(q_{2}\right)$ satisfies the equation, then $\mathcal{M}\left(q_{2}+\delta u\right)$ also does, because

$$
\mathcal{M}\left(q_{2}+\delta u\right)=\int_{k_{2}} \mathcal{K}\left(q_{2}+\delta u-k_{2}\right) \mathcal{M}\left(k_{2}\right)=\int_{k_{2}} \mathcal{K}\left(q_{2}-k_{2}\right) \mathcal{M}\left(k_{2}+\delta u\right)
$$

Therefore $\mathcal{M}\left(q_{2}\right)$ and $\mathcal{M}\left(q_{2}+\delta u\right)$ characterize the same eigen-states. These degenerated states are labeled by the aforementioned momentum $P_{1}$ in the following way. These two states are different from each other only by a constant phase,

$$
\mathcal{M}\left(q_{2}+\delta u\right)=\mathrm{e}^{\mathrm{i} C_{\delta u}} \mathcal{M}\left(q_{2}\right)
$$

On the other hand, one can write

$$
\mathcal{M}\left(q_{2}+\delta u\right)=\mathrm{e}^{\delta u \partial_{q_{2}}} \mathcal{M}\left(q_{2}\right)
$$

so that the solution is found to be

$$
\mathcal{M}\left(q_{2}\right)=\mathrm{e}^{\mathrm{i} q_{2} C_{\delta u} / \delta u} \mathcal{M}\left(q_{2}=0\right)
$$

The constant factor $C_{\delta u} / \delta u$ characterizes the amplitude and spectrum of the bound state. Now, using a fictious transverse momentum $P_{1}=-C_{\delta u} / \delta u$, we may label the states connected by the translation (40) as

$$
\mathcal{M}_{P_{1}}\left(q_{2}\right) \equiv \mathrm{e}^{-\mathrm{i} q_{2} P_{1} / B_{f}} \mathcal{M}_{P_{1}}\left(q_{2}=0\right)
$$

This relation indicates that the shift of the relative momentum $q_{2}$ affects on neither the total momenta $P_{1}$ nor $P_{2}$. Substituting the above expression for the previous symbolic form, one finds

$$
\mathcal{M}_{P_{1}}\left(q_{2}=0\right)=\int_{k_{2}} \mathcal{K}\left(q_{2}-k_{2}\right) \mathrm{e}^{\mathrm{i}\left(q_{2}-k_{2}\right) P_{1} / B_{f}} \mathcal{M}_{P_{1}}\left(k_{2}=0\right)
$$


This new phase factor for $P_{1}$ can be combined with the factor from the Schwinger phase, yielding

$$
-\mathrm{i}\left(q_{1}-k_{1}\right) \frac{P_{2}}{B_{f}}+\mathrm{i}\left(q_{2}-k_{2}\right) \frac{P_{1}}{B_{f}}=\mathrm{i} \frac{\vec{B}_{f} \times \vec{P}_{\perp}}{B_{f}^{2}} \cdot(\vec{q}-\vec{k})_{\perp} \equiv \mathrm{i} \vec{\xi}_{P}^{f} \cdot(\vec{q}-\vec{k})_{\perp},
$$

which is rotationally symmetric with respect to $\vec{P}_{\perp}$. Now recovering all the other indices in the original expression (32), the Bethe-Salpeter equation can be written in the dimensionally reduced form

$$
\mathcal{M}_{P_{\perp} P_{3}}^{f}\left(q_{L}\right)=-S^{f}\left(q_{L}^{+}\right) \gamma_{L}\left[\int_{k_{L}} \mathcal{W}_{L L^{\prime}}^{f}\left(q_{L}-k_{L} ; P_{\perp}\right) \mathcal{M}_{P_{\perp} P_{3}}^{f}\left(k_{L}\right)\right] \gamma_{L^{\prime}} S^{f}\left(q_{L}^{-}\right)
$$

where $\mathcal{M}_{P_{\perp} P_{3}}\left(q_{L}\right) \equiv \mathcal{M}_{P_{\perp} P_{3}}\left(q_{2}=0, q_{L}\right)=\mathrm{e}^{\mathrm{i} q_{2} P_{1} / B_{f}} \mathcal{M}_{P_{\perp} P_{3}}\left(q_{2}, q_{L}\right)$, and we have defined the $(1+1)$ dimensional force

$$
\mathcal{W}_{L L^{\prime}}^{f}\left(q_{L}-k_{L} ; P_{\perp}\right) \equiv C_{F} \int_{k_{\perp}} D_{L L^{\prime}}(q-k)\left[\mathcal{R}_{00}^{f}\left(\vec{q}_{\perp}-\vec{k}_{\perp}\right)\right]^{2} \times \mathrm{e}^{\mathrm{i} \vec{\xi}_{P}^{f} \cdot\left(\vec{q}_{\perp}-\vec{k}_{\perp}\right)}
$$

To get some insights on the impact of the quantum phase, it is instructive to look at the expression in the coordinate space. With the inverse Fourier transform, the above expression becomes

$$
\mathcal{W}_{L L^{\prime}}^{f}\left(r_{L} ; P_{\perp}\right)=C_{F} \frac{\left|B_{f}\right|}{2 \pi} \int_{\vec{r}_{\perp}} D_{L L^{\prime}}\left(r_{L}, \vec{r}_{\perp}\right) \mathrm{e}^{-\frac{\left|B_{f}\right|}{2}\left(\vec{r}_{\perp}-\vec{\xi}_{P}^{f}\right)^{2}}
$$

If the variation in $D_{L L^{\prime}}$ is much milder than in $\mathrm{e}^{-\frac{\left|B_{f}\right|}{2}\left(\vec{r}_{\perp}-\vec{\xi}_{P}^{f}\right)^{2}}$, we can use a factorization to get

$$
\mathcal{W}_{L L^{\prime}}^{f}\left(r_{L} ; P_{\perp}\right) \simeq C_{F} D_{L L^{\prime}}\left(r_{L}, \vec{r}_{\perp}=\vec{\xi}_{P}^{f}\right), \quad \frac{\left|B_{f}\right|}{2 \pi} \int_{\vec{r}_{\perp}} \mathrm{e}^{-\frac{\left|B_{f}\right|}{2}\left(\vec{r}_{\perp}-\vec{\xi}_{P}^{f}\right)^{2}}=1
$$

where we have replaced the $\vec{r}_{\perp}$ in the potential by $\vec{\xi}_{P}$, and then carried out the integration. As an important example, we find that the linear rising potential results in

$$
\mathcal{W}_{00}^{f}\left(r_{L} ; P_{\perp}\right) \sim \sigma \sqrt{r_{3}^{2}+\left(\vec{\xi}_{P}^{f}\right)^{2}}
$$

For such an interaction, $\vec{\xi}_{P}^{f}$ can be dropped off in the limit of large $B \gg P_{2}$, and the problem is reduced to the bound state problem with a potential $\sim \sigma\left|r_{3}\right|$ in one spatial dimension; in this case the bound states are squeezed into the one-dimension shape and their spectra are independent of $B$.

The above qualitative picture of the bound states will be considerably changed if the mutual interaction has short-range nature where our factorization approximation may not be valid. For a contrast example, a contact interaction gives the following one-dimensional potential,

$$
D_{\text {contact }}(\vec{r}) \sim-\Lambda_{\mathrm{QCD}}^{-2} \delta(\vec{r}) \quad \rightarrow \quad \mathcal{W}_{\text {contact }}\left(r_{3} ; P_{\perp}\right) \simeq-\frac{\left|B_{f}\right|}{\Lambda_{\mathrm{QCD}}^{2}} \mathrm{e}^{-\frac{P_{\perp}^{2}}{2\left|B_{f}\right|}} \delta\left(r_{3}\right)
$$


which strongly depends on $B$ and the binding energy grows as $B$ increases. If one uses another interaction, e.g., $D \sim 1 / \sqrt{r_{3}^{2}+\vec{r}_{\perp}^{2}}$, such a strong $B$-dependence would be tempered. However, in this case, some special care is necessary for the $r_{3}^{2} \sim 0$ region where the $r_{\perp}$-dependence and thereby $\xi_{P}$-dependence again become significant. For these cases, details of $\vec{\xi}_{P}^{f}$ become very important, and the spectra will be sensitive to the value of $B$. Having compared the bound states by three simple interactions, we find that the bound state spectra are strongly model-dependent, and especially depend on whether the interactions have short- or long-range nature.

Finally we shall examine the structure of the equal-time amplitude in the coordinate space. Following from Eqs. (27) and (41), we find the coordinate-space expression as

$$
\mathcal{M}_{P_{\perp} P_{3}}^{f}(\vec{r} ; \vec{R}) \equiv \mathcal{N} \mathrm{e}^{\mathrm{i}\left(P_{2} R_{2}+P_{3} R_{3}\right)} \int_{q_{2}} \mathrm{e}^{\mathrm{i} q_{2} r_{2}} \mathcal{H}_{0}^{f}\left(x_{1}-r_{q_{2}^{+}}^{f}\right) \mathcal{H}_{0}^{f}\left(x_{1}^{\prime}-r_{q_{2}^{-}}^{f}\right) \mathrm{e}^{-\mathrm{i} q_{2} P_{1} / B_{f}} \mathcal{M}_{P_{\perp} P_{3}}^{f}\left(r_{3}\right)
$$

where the inverse Fourier transform of $\mathcal{M}\left(q_{3}\right)$ is denoted as a normalized one-dimensional wavefunction $\mathcal{M}_{P_{\perp} P_{3}}^{f}\left(r_{3}\right)$. After carrying out the $q_{2}$-integration, we arrive at

$$
\mathcal{M}_{P_{\perp} P_{3}}^{f}(\vec{r} ; \vec{R})=\frac{\mathrm{e}^{\mathrm{i} \vec{P} \cdot \vec{R}}}{\sqrt{V_{3}}} \times \mathrm{e}^{-\mathrm{i} B_{f} R_{1} r_{2}} \times \sqrt{\frac{\left|B_{f}\right|}{2 \pi}} \mathrm{e}^{-\frac{\left|B_{f}\right|}{4}\left(\vec{r}_{\perp}-\vec{\xi}_{P}^{f}\right)^{2}} \mathcal{M}_{P_{\perp} P_{3}}^{f}\left(r_{3}\right),
$$

with $V_{3}$ being the three-dimensional volume. Several remarks are in order: (i) The first factor is the plane wave part characterized by conserved momenta, $\vec{P}=\left(P_{1}, P_{2}, P_{3}\right)$. A factor of $\mathrm{e}^{\mathrm{i} P_{1} R_{1}}$ for $P_{1}$ was obtained after integrating over $q_{2}$. (ii) The Schwinger phase appears in the second factor because our interpolating fermion fields are separated in distance; if we started with a manifestly gauge invariant operator, $\bar{\chi}(x) \mathrm{e}^{\mathrm{i} e \int_{y}^{x} \mathrm{~d} \vec{s} \cdot \vec{A}(s)} \chi(y)$, then the Schwinger phase could be eliminated. (iii) The third factor is the Gaussian function which characterizes the wavefunction for the relative transverse coordinate. The average transverse distance between the quark and anti-quark is given by $\vec{\xi}_{P}^{f} \sim B_{f}^{-1}$, which is vanishing as a magnitude of $B$ increases, again indicating the squeezed bound state.

\section{Charged mesons : the $e_{f} \neq e_{f^{\prime}}$ case}

Next we turn to the $e_{f} \neq e_{f^{\prime}}$ case. Since the COM and relative motions are correlated, the dynamics is more involved than the $e_{f}=e_{f^{\prime}}$ case in the last section. First we find the quantum phase (34) as

$$
\left[\Xi_{q_{2}^{+}, k_{2}^{+}}^{f}-\Xi_{q_{2}^{-}, k_{2}^{-}}^{f^{\prime}}\right]\left(q_{1}-k_{1}\right)=-a\left(q_{1}-k_{1}\right)\left[\left(q_{2}+\zeta P_{2}\right)+\left(k_{2}+\zeta P_{2}\right)\right]
$$

where we introduced

$$
a=\frac{B_{f^{\prime}}-B_{f}}{2 B_{f^{\prime}} B_{f}}, \quad \zeta=\frac{1}{2} \frac{B_{f^{\prime}}+B_{f}}{B_{f^{\prime}}-B_{f}} .
$$


Note that, when the relative momentum is $q_{2}-\zeta P_{2}$, the $P_{2}$-dependence in the exponent is absorbed by the Bethe-Salpeter amplitude, i.e., the Bethe-Salpeter equation (32) can be rewritten as

$$
\begin{aligned}
\mathcal{M}_{P_{2} P_{3}}^{f f^{\prime}}\left(q_{2}-\zeta P_{2}, q_{L}\right)=(-\mathrm{i})^{2} C_{F} S^{f}\left(q_{L}^{+}\right) \gamma_{L} \int_{k_{L}, k_{\perp}} D_{L L^{\prime}}(q-k) \mathcal{R}_{00}^{f} \mathcal{R}_{00}^{f^{\prime}}\left(\vec{q}_{\perp}-\vec{k}_{\perp}\right) \mathrm{e}^{-\mathrm{i} a\left(q_{1}-k_{1}\right)\left(q_{2}+k_{2}\right)} \\
\times \mathcal{M}_{P_{2} P_{3}}^{f f^{\prime}}\left(k_{2}-\zeta P_{2}, k_{L}\right) \gamma_{L^{\prime}} S^{f^{\prime}}\left(q_{L}^{-}\right)
\end{aligned}
$$

It is important to notice that the $P_{2}$-dependence appears only through the amplitude function $\mathcal{M}$; the other part is $P_{2}$-independent, meaning that the amplitudes at $P_{2}=0$ and $P_{2} \neq 0$ obey the same equation. Therefore the solutions at $P_{2}=0$ and $P_{2} \neq 0$ are physically equivalent, and are equal modulo some constant phase factor (here we may regard $P_{2}$ as a constant because it is conserved):

$$
\mathcal{M}_{P_{2} P_{3}}^{f f^{\prime}}\left(q_{2}-\zeta P_{2}, q_{L}\right)=\mathrm{e}^{\mathrm{i} C_{P_{2}}} \mathcal{M}_{P_{2}=0, P_{3}}^{f f^{\prime}}\left(q_{2}, q_{L}\right)
$$

We find that the COM and relative motions are correlated because the shift of $q_{2}$ affects on the $P_{2}$, and vice versa. This contrasts to the neutral case discussed around Eq. (41).

To get solutions for all $P_{2}$, we have only to analyze the Bethe-Salpeter equation at $P_{2}=0$ and use the above relation. Therefore from now on we consider the $P_{2}=0$ case and shall drop off the subscript $P_{2}$. The remaining calculations are worked out as follows. Taking the coordinate-space expression of the propagator and then carrying out the integration over $k_{1}$, we arrive at

$$
\mathcal{M}_{P_{3}}^{f f^{\prime}}\left(q_{2}, q_{L}\right)=(-\mathrm{i})^{2} S^{f}\left(q_{L}^{+}\right) \gamma_{L}\left[\int_{k_{L}} \int_{r_{L}} \mathrm{e}^{-\mathrm{i}\left(q_{L}-k_{L}\right) r_{L}}(\mathcal{K} \mathcal{M})_{P_{3}}^{f f^{\prime}}\right] \gamma_{L^{\prime}} S^{f^{\prime}}\left(q_{L}^{-}\right)
$$

where $\mathcal{K}$ assembles the transverse part,

$$
(\mathcal{K} \mathcal{M})_{P_{3}}^{f f^{\prime}}=\sqrt{4 b \pi} C_{F} \int_{\vec{r}_{\perp}} \int_{k_{2}} D_{L L^{\prime}}\left(r_{L}, \vec{r}_{\perp}\right) \mathrm{e}^{-b\left[r_{1}+a\left(q_{2}+k_{2}\right)\right]^{2}} \mathrm{e}^{-\mathrm{i}\left(q_{2}-k_{2}\right) r_{2}-\frac{1}{4 b}\left(q_{2}-k_{2}\right)^{2}} \mathcal{M}_{P_{3}}^{f f^{\prime}}\left(k_{2}, k_{L}\right) .
$$

In addition to $a \sim B^{-1}$ introduced above, we have

$$
b=\frac{\left|B_{f^{\prime}} B_{f}\right|}{\left|B_{f^{\prime}}\right|+\left|B_{f}\right|}=\mathcal{O}(B)
$$

To proceed to further analytic computation, we focus on long-range interactions such as the linear rising potential, and make several approximations suitable for the analysis of those important models. (i) Below we assume that the propagator varies much more slowly than the Gaussian $\mathrm{e}^{-b\left[r_{1}+a\left(k_{2}+q_{2}\right)\right]^{2}}$, which tends to be the case in strong magnetic fields. Then we can apply a factorization,

$$
\int_{r_{1}} D_{L L^{\prime}}\left(r_{L}, \vec{r}_{\perp}\right) \mathrm{e}^{-b\left[r_{1}+a\left(q_{2}+k_{2}\right)\right]^{2}} \simeq D_{L L^{\prime}}\left(r_{L}, r_{1}=-a\left(q_{2}+k_{2}\right), r_{2}\right) \times \sqrt{\frac{\pi}{b}}
$$


where $r_{1}$ is replaced by the center of the peak, $-a\left(q_{2}+k_{2}\right) \sim-\left(q_{2}+k_{2}\right) / B$. (ii) Assuming a longrange interaction, that is dominated by small momentum transfer processes $\left|q_{2}-k_{2}\right| \sim \Lambda_{\mathrm{QCD}} \ll$ $|B|^{1 / 2}$, we find

$$
\mathrm{e}^{-\frac{1}{4 b}\left(q_{2}-k_{2}\right)^{2}} \simeq 1, \quad a\left(q_{2}+k_{2}\right) \sim 2 a k_{2}+O\left(\Lambda_{\mathrm{QCD}} / B\right)
$$

so that Eq. (57) reads

$$
(\mathcal{K} \mathcal{M})_{P_{3}}^{f f^{\prime}} \simeq 2 \pi C_{F} \int_{r_{2}} \mathrm{e}^{-\mathrm{i} q_{2} r_{2}} \int_{k_{2}} D_{L L^{\prime}}\left(r_{L}, r_{1}=-2 a k_{2}, r_{2}\right) \mathrm{e}^{\mathrm{i} k_{2} r_{2}} \mathcal{M}_{P_{3}}^{f f^{\prime}}\left(k_{2}, k_{L}\right)
$$

Now that the $q_{2}$-dependence was factorized in the exponential factor, our Bethe-Salpeter equation, in the coordinate representation with respect to $r_{2}$, is obtained as

$$
\begin{aligned}
\mathcal{M}_{P_{3}}^{f f^{\prime}}\left(r_{2}, q_{L}\right) & \simeq(-\mathrm{i})^{2} S^{f}\left(q_{L}^{+}\right) \gamma_{L} \int_{k_{L}} \int_{r_{L}} \mathrm{e}^{-\mathrm{i}\left(q_{L}-k_{L}\right) r_{L}} 2 \pi C_{F} \\
& \times\left[\int_{k_{2}} D_{L L^{\prime}}\left(r_{L}, r_{1}=-2 a k_{2}, r_{2}\right) \mathrm{e}^{\mathrm{i} k_{2} r_{2}} \mathcal{M}_{P_{3}}^{f f^{\prime}}\left(k_{2}, k_{L}\right)\right] \gamma_{L^{\prime}} S^{f^{\prime}}\left(q_{L}^{-}\right) .
\end{aligned}
$$

This approximate form is valid for long-range interaction models whose spatial variations in the coordinate $r_{1}$ is much milder than the Gaussian form factor $\sim \mathrm{e}^{-|B| r_{1}^{2}}$.

The expression (62) has a somewhat involved structure, and requires some heuristic explanations. Because we are considering the low energy spectra in confining theories, the average $|\vec{r}|$ should be $\mathcal{O}\left(\Lambda_{\mathrm{QCD}}^{-1}\right)$. Especially, $\left\langle r_{3}\right\rangle$ cannot be much smaller than $\Lambda_{\mathrm{QCD}}^{-1}$ as in the case of $B=0$, because of the kinetic energy in the $x_{3}$-direction. However, as illustrated in Fig. 1, the transverse size, $\left\langle r_{1}\right\rangle$ and $\left\langle r_{2}\right\rangle$, can be much smaller than $\Lambda_{\mathrm{QCD}}^{-1}$. When one of the coordinates $\left\langle r_{1}\right\rangle$, that is $\sim a\left\langle k_{2}\right\rangle$ in Eq. (62), is much smaller than $\Lambda_{\mathrm{QCD}}^{-1}$, the wavefunction should strongly damp beyond $k_{2} \sim|B| / \Lambda_{\mathrm{QCD}}$. On the other hand, when $\left|\left\langle r_{2}\right\rangle\right|$ is small, the wavefunction should contain the Fourier components from zero to values much larger than $\Lambda_{\mathrm{QCD}}$; otherwise, i.e., if only soft components $\left(\lesssim \Lambda_{\mathrm{QCD}}\right)$ are included, we can get only $\left|\left\langle r_{2}\right\rangle\right|>\Lambda_{\mathrm{QCD}}^{-1}$. These two observations indicate that, for low energy states, the damping scale, $\Lambda_{\text {damp }}$, of the transverse wavefunction should satisfy a hierarchy

$$
\Lambda_{\mathrm{QCD}} \ll \Lambda_{\mathrm{damp}} \ll|B| / \Lambda_{\mathrm{QCD}}, \quad \mathcal{M}\left(k_{2}, k_{L}\right) \sim 0 \quad\left(\text { for }\left|k_{2}\right| \gg \Lambda_{\mathrm{damp}}\right) .
$$

Furthermore, note that the relative distance in the transverse direction, $\left\langle r_{\perp}\right\rangle$, is minimized when

$$
\left\langle r_{\perp}^{2}\right\rangle \sim\left\langle k_{2}^{2}\right\rangle / B^{2}+\left\langle r_{2}^{2}\right\rangle \geq 2\left(\left\langle k_{2}^{2}\right\rangle\left\langle r_{2}^{2}\right\rangle\right)^{1 / 2} /|B|
$$

where the equality holds when $\left\langle r_{2}^{2}\right\rangle=\left\langle k_{2}^{2}\right\rangle / B^{2}$. This relation is satisfied when $\left\langle k_{2}^{2}\right\rangle \sim 1 /\left\langle r_{2}^{2}\right\rangle \sim|B|$, and such expectation values can be obtained for wavefunctions such as $\mathcal{M}\left(k_{2}\right) \sim \mathrm{e}^{-k_{2}^{2} /|B|}$. Such 
a solution with $\left\langle r_{1}^{2}\right\rangle \sim\left\langle r_{2}^{2}\right\rangle \sim|B|^{-1}$ is a natural candidate for the ground state because $\sim\left\langle r_{\perp}^{2}\right\rangle$ is the minimal area for the circular motion that was expected from the classical picture in Fig.5 (right). At $|B| \gg \Lambda_{\mathrm{QCD}}^{2}$, charged mesons at low energy are squeezed in a nearly one dimensional shape. For the $n_{\perp}$-th excited states, the transverse area grows as $\sim\left\langle r_{\perp}^{2}\right\rangle_{n_{\perp}} \sim n_{\perp}|B|^{-1}$. Therefore the transverse extension is negligible until $n_{\perp}$ reaches $\sim|B| / \Lambda_{\mathrm{QCD}}^{2}$, and there are many squeezed charged mesons at low energy.

\subsection{Example 1: Confining harmonic oscillator}

We examine an instantaneous potential of the harmonic oscillator. Using this solvable model, we learn basic structure of the low energy bound state, and extend it to analysis of more general potentials. The explicit form of the potential is given by

$$
D_{L L^{\prime}}^{\text {harmo }}(r)=\delta_{L 0} \delta_{L^{\prime} 0} \delta\left(r_{0}\right) D^{\text {harmo }}(\vec{r}), \quad D^{\text {harmo }}(\vec{r})=\frac{c_{H} \Lambda_{\mathrm{QCD}}^{3}}{2 \pi C_{F}}\left(r_{3}^{2}+r_{\perp}^{2}\right)
$$

where $c_{H}$ is some dimensionless constant. In this model, we can rewrite the transverse part of the Bethe-Salpeter equation (62) as

$$
\int_{k_{2}}\left(r_{3}^{2}+\frac{k_{2}^{2}}{|B|^{2}}+r_{2}^{2}\right) \mathrm{e}^{\mathrm{i} k_{2} r_{2}} \mathcal{M}_{P_{3}}^{f f^{\prime}}\left(k_{2}, k_{L}\right)=\left(r_{3}^{2}-\frac{\partial_{2}^{2}}{|B|^{2}}+r_{2}^{2}\right) \mathcal{M}_{P_{3}}^{f f^{\prime}}\left(r_{2}, k_{L}\right) .
$$

The above operator can be diagonalized by using the harmonic oscillator bases; for the $n_{\perp^{-}}$ th excited state in the transverse directions, the corresponding wavefunction is found to be $\mathcal{M}_{n_{\perp}}\left(r_{2}, r_{L}\right)=\mathcal{M}_{n_{\perp}}\left(r_{L}\right) \mathcal{H}_{n_{\perp}}\left(r_{2}\right)$, e.g., for the ground state, $\mathcal{M}_{0}\left(r_{2}, r_{L}\right)=\mathcal{M}_{0}\left(r_{L}\right) \mathrm{e}^{-\frac{|B|}{2} r_{2}^{2}}$. Making use of these bases, the transverse dynamics is solved as

$$
\left(r_{3}^{2}-\frac{\partial_{2}^{2}}{|B|^{2}}+r_{2}^{2}\right) \mathcal{M}_{n_{\perp}}\left(r_{2}, k_{L}\right)=\mathcal{H}_{n_{\perp}}\left(r_{2}\right)\left(r_{3}^{2}+\frac{2 n_{\perp}+1}{|B|}\right) \mathcal{M}_{n_{\perp}}\left(k_{L}\right) .
$$

Inserting this expression into Eq. (62), the Bethe-Salpeter equation reads

$$
\mathcal{M}_{n_{\perp}, P_{3}}^{f f^{\prime}}\left(q_{3}\right) \simeq(-\mathrm{i})^{2} \int_{q_{0}} S^{f}\left(q_{L}^{+}\right) \gamma_{0}\left[\int_{k_{3}} D_{n}^{\text {harmo }}\left(q_{3}-k_{3}\right) \mathcal{M}_{n_{\perp}, P_{3}}^{f f^{\prime}}\left(k_{3}\right)\right] \gamma_{0} S^{f^{\prime}}\left(q_{L}^{-}\right)
$$

where, for the $n_{\perp}$-th excited transverse modes,

$$
D_{n_{\perp}}^{\text {harmo }}\left(q_{3}\right)=c_{H} \Lambda_{\mathrm{QCD}}^{3} \int_{r_{3}} \mathrm{e}^{\mathrm{i} q_{3} r_{3}}\left(r_{3}^{2}+\frac{2 n_{\perp}+1}{|B|}\right) .
$$

Since the instantaneous potential is independent of the temporal components, the $q_{0}$-integral results in the equal-time amplitude, $\mathcal{M}\left(q_{3}\right)=\int_{q_{0}} \mathcal{M}\left(q_{L}\right)$. In Eq. (69), the second term, $\left(2 n_{\perp}+\right.$ $1) /|B|$, is irrelevant until the integer $n_{\perp}$ reaches $\sim|B| / \Lambda_{\mathrm{QCD}}^{2}$. Therefore, for $n_{\perp} \lesssim|B| / \Lambda_{\mathrm{QCD}}^{2}$, our problem is reduced to a one-dimensional bound state problem with respect to the relative

coordinate $r_{3}$. This result indicates that there are a lot of (nearly) degenerated bound states at low energy whose energies are determined solely by the one-dimensional dynamics in the $x_{3^{-}}$ direction. 


\subsection{Example 2: Confining linear rising potential}

Next we proceed to a more realistic confining potential, that is, an instantaneous linear rising potential

$$
D_{L L^{\prime}}^{\text {linear }}(r)=\delta_{L 0} \delta_{L^{\prime} 0} \delta\left(r_{0}\right) D^{\text {linear }}(\vec{r}), \quad D^{\text {linear }}(\vec{r})=\frac{\sigma}{2 \pi C_{F}} \sqrt{r_{3}^{2}+r_{\perp}^{2}},
$$

with the string tension $\sigma \sim \Lambda_{\mathrm{QCD}}^{2}$. We shall again analyze the transverse part in Eq. (62),

$$
\int_{k_{2}} \sqrt{r_{3}^{2}+\frac{k_{2}^{2}}{|B|^{2}}+r_{2}^{2}} \mathrm{e}^{\mathrm{i} k_{2} r_{2}} \mathcal{M}_{P_{3}}^{f f^{\prime}}\left(k_{2}, k_{L}\right)
$$

While it is tempting to replace $k_{2}$ by $-\mathrm{i} \partial_{2}$ as before, this is not a legitimate treatment since $\partial_{2}$ and $r_{2}$ inside the square-root do not commute. Thus the analysis is more involved than the harmonic oscillator case.

Nevertheless, we may use an approximation and extend the procedure examined in the last subsection to analyze low energy states which satisfy the relation $\left|\left\langle r_{\perp}\right\rangle\right| \lesssim|B|^{-1 / 2} \ll\left|\left\langle r_{3}\right\rangle\right| \sim \Lambda_{\mathrm{QCD}}^{-1}$ discussed around Eq. (64). We also note that the potential energy becomes important only when $\left|\left\langle r_{3}\right\rangle\right| \gtrsim \Lambda_{\mathrm{QCD}}^{-1}$, otherwise the potential energy term is simply negligible. In such a domain of $r_{3}$ and $r_{\perp}$, we can expand the potential as

$$
\sim \int_{k_{2}}\left|r_{3}\right|\left[1+\frac{1}{2\left|r_{3}^{2} B\right|}\left(\frac{k_{2}^{2}}{|B|}+|B| r_{2}^{2}\right)+O\left(1 /\left|r_{3}^{2} B\right|^{2}\right)\right] \mathrm{e}^{\mathrm{i} k_{2} r_{2}} \mathcal{M}_{P_{3}}^{f f^{\prime}}\left(k_{2}, k_{L}\right),
$$

where the expression is valid only for $\left|r_{3}\right| \gg|B|^{-1 / 2}$. In the second term, we can make replacement $k_{2} \rightarrow-\mathrm{i} \partial_{2}$, and diagonalize it by using the harmonic oscillator bases. Corrections to these leading terms are of the order of $\left(\Lambda_{\mathrm{QCD}}^{2} /|B|\right)^{2} \ll 1$. Picking up a class of the higher order corrections, one can replace $\sqrt{r_{3}^{2}+k_{2}^{2} /|B|+r_{2}^{2}}$ by $\sqrt{r_{3}^{2}-\partial_{2}^{2} /|B|+r_{2}^{2}}+O\left(\Lambda_{\mathrm{QCD}}^{4} /|B|^{2}\right)$. Within this precision level, one can expand $\mathcal{M}\left(q_{2}\right)$ by the harmonic oscillator bases, and obtains

$$
\mathcal{M}_{n_{\perp}, P_{3}}^{f f^{\prime}}\left(q_{3}\right) \simeq(-\mathrm{i})^{2} \int_{q_{0}} S^{f}\left(q_{L}^{+}\right) \gamma_{0}\left[\int_{k_{3}} D_{n_{\perp}}^{\text {linear }}\left(q_{3}-k_{3}\right) \mathcal{M}_{n_{\perp}, P_{3}}^{f f^{\prime}}\left(k_{3}\right)\right] \gamma_{0} S^{f^{\prime}}\left(q_{L}^{-}\right)
$$

where the one-dimensional potential is given by

$$
D_{n_{\perp}}^{\text {linear }}\left(q_{3}\right)=\sigma \int_{\left|r_{3}\right| \gg|B|^{-1 / 2}} \mathrm{e}^{\mathrm{i} q_{3} r_{3}} \sqrt{r_{3}^{2}+\frac{2 n_{\perp}+1}{|B|}}+O\left(\Lambda_{\mathrm{QCD}}^{4} /|B|^{2}\right) .
$$

As in the harmonic oscillator model, the spectra are governed by the longitudinal dynamics until $n_{\perp}$ reaches $\sim|B| / \Lambda_{\mathrm{QCD}}^{2}$, so that there are many low-energy states with the energies $\sim \Lambda_{\mathrm{QCD}}$. While the square root induces the mixing between different harmonic oscillator bases, the overall tendencies are the same as in the result of the harmonic oscillator model. In fact, the above observation suggest that, as far as the above expansion is valid, the large degeneracy of the low energy bound states is a universal feature of long-range interaction models. 


\section{Meson resonance gas at finite temperature: percolation and chiral restoration}

In the last sections we examined the properties of the low-energy bound states on the basis of the Bethe-Salpeter equations, and obtained the proper quantum numbers to specify their states. Especially, we discussed the difference between the neutral and charged mesons, and also the large degeneracy in the low energy domain. Without elaborating quantitative aspects of the meson structures, one can readily make a number of qualitative statements on the thermal partition function by using only the results obtained in the last sections.

In this section we first examine the density of states of mesons, and then consider the thermodynamic partition function for the $q \bar{q}$ sector. We address how the percolation and chiral restoration proceed as temperature increases in the presence of strong magnetic fields.

\subsection{Density of states}

As a warming up, we begin with the review of the density of states for a quark, and then consider those for neutral and charged mesons. Below we concentrate on the density of states in the transverse directions as the continuous longitudinal spectrum is the same as in $B=0$ case. To count the number of a charged fermion state in magnetic fields, we will use a periodic lattice with the lattice spacing $a=2 \pi \Lambda_{\mathrm{UV}}^{-1}$ and with the lengths $\left(L_{1}, L_{2}\right)$. These results provides the integral measures of the partition functions in the next subsection.

\subsubsection{Density of states for single quarks}

Without magnetic fields, the total number of states for single fermions is as usual given by

$$
\frac{L_{1} L_{2}}{a^{2}} \sim \sum_{n_{1}}^{L_{1} / a} \sum_{n_{2}}^{L_{2} / a} \sim \frac{L_{1} L_{2}}{(2 \pi)^{2}} \int^{\Lambda_{\mathrm{UV}}} \mathrm{d} p_{1} \int^{\Lambda_{\mathrm{UV}}} \mathrm{d} p_{2},
$$

This is nothing but the number of sites, and we converted it into the continuum expression. (We do not take into account non-integer part of $L / a$ and spins.) In the presence of magnetic fields, these states are divided into several LLs. In the Landau gauge, only momentum $p_{2}=2 \pi n_{2} / L_{2}$ $\left(n_{2}\right.$ : integer) is conserved, so that a fermion state in the transverse dynamics is labeled by $p_{2}$ and the index of the Landau level, $n_{L}$. Also, since the location of the fermion is given by $R_{1}=p_{2} / B$, this coordinate should be smaller than the length $L_{1}$, leading to the following condition on the maximum number of $n_{2}$ :

$$
0 \leq \frac{2 \pi n_{2}}{|B| L_{2}} \leq L_{1} \quad \rightarrow \quad n_{2}^{\max } \sim \frac{|B| L_{1} L_{2}}{2 \pi},
$$

which tells us the number of states for each LL. Then the sum of states can be written as

$$
\frac{L_{1} L_{2}}{a^{2}} \sim \sum_{n_{L}=0}^{2 \pi /|B| a^{2}} \times \sum_{n_{2}=0}^{|B| L_{1} L_{2} / 2 \pi} \sim \sum_{n_{L}=0}^{\Lambda_{\mathrm{UV}}^{2} /(2 \pi|B|)} \frac{L_{2}}{2 \pi} \int_{0}^{|B| L_{1}} \mathrm{~d} p_{2} .
$$


To determine the maximum of $n_{L}$, we divided the total number of states $\sim L_{1} L_{2} / a^{2}$ by the number of states in each Landau level, $\sim|B| L_{1} L_{2}$. In most cases quantities, that we study, are independent of $p_{2}$, and we can carry out the integral over $p_{2}$. Dividing the number of states by the system volume $L_{1} L_{2}$, we find the well-known degeneracy factor $|B| / 2 \pi$ for each LL. .

\subsubsection{Density of states for mesons}

Without magnetic fields, the total number of meson states are given by

$$
\left(\frac{L_{1} L_{2}}{a^{2}}\right)^{2} \sim \sum_{n_{1}}^{L_{1} / a} \sum_{n_{2}}^{L_{2} / a} \sum_{n_{1}^{\prime}}^{L_{1} / a} \sum_{n_{2}^{\prime}}^{L_{2} / a} \sim \frac{L_{1} L_{2}}{(2 \pi)^{2}} \int^{\Lambda_{\mathrm{UV}}} \mathrm{d} \vec{P}_{\perp} \times \sum_{N_{b}=0}^{L_{1} L_{2} / a^{2}}
$$

where we have reorganized the sum into the total and relative momenta, and $N_{b}$ labels bound states. Note that there is a maximum for the number of bound states, $N_{b}^{\max }=L_{1} L_{2} / a^{2}$, reflecting the condition that the size of meson should be much smaller than the system size, or much larger than the lattice spacing.

In the presence of magnetic fields, the sum is decomposed as

$$
\left(\frac{L_{1} L_{2}}{a^{2}}\right)^{2} \sim \sum_{n_{L}=0}^{2 \pi /|B| a^{2}} \sum_{n_{L}^{\prime}=0}^{2 \pi /|B| a^{2}} \times \sum_{n_{2}=0}^{|B| L_{1} L_{2} / 2 \pi} \sum_{n_{2}^{\prime}=0}^{|B| L_{1} L_{2} / 2 \pi} .
$$

Now we focus on the meson states made only of LLLs. For such mesons with $n_{L}=n_{L}^{\prime}=0$, the maximum number of states on the lattice is $\sim\left(|B| L_{1} L_{2}\right)^{2}$. As in the $B=0$ case, we can reorganize the summation of $n_{2}$ and $n_{2}^{\prime}$ as the summation for conserved total momenta, $n_{2}^{G}=n_{2}+n_{2}^{\prime}$, and relative momenta $\delta n_{2}=n_{2}-n_{2}^{\prime}$ as

$$
\left(\frac{|B| L_{1} L_{2}}{2 \pi}\right)^{2} \sim \sum_{n_{2}^{G}=0}^{|B| L_{1} L_{2} / 2 \pi} \times \sum_{\delta n_{2}=0}^{|B| L_{1} L_{2} / 2 \pi} \sim \frac{L_{2}}{2 \pi} \int_{0}^{|B| L_{1}} \mathrm{~d} P_{2} \times \sum_{\delta n_{2}=0}^{|B| L_{1} L_{2} / 2 \pi} .
$$

Depending on whether meson states are neutral or charged, the summation over $\delta n_{2}$ is rearranged in two different ways below.

For neutral mesons, we have seen that, in addition to $P_{2}$, one can define another conserved momentum that is called $P_{1}$. The momentum $P_{1}$ is also continuous and specifies the location of mesons in the $x_{2}$-direction as $P_{2}$ does in the $x_{1}$-direction. Therefore we have

$$
\left(\frac{|B| L_{1} L_{2}}{2 \pi}\right)^{2} \sim \frac{L_{2}}{2 \pi} \int_{0}^{|B| L_{1}} \mathrm{~d} P_{2} \times \frac{L_{1}}{2 \pi} \int_{0}^{|B| L_{2}} \mathrm{~d} P_{1} \quad \text { (neutral mesons). }
$$

On the other hand, the charged mesons have discrete spectra instead of the continuous $P_{1}$, so that the sum is expressed as

$$
\left(\frac{|B| L_{1} L_{2}}{2 \pi}\right)^{2} \sim \frac{L_{2}}{2 \pi} \int_{0}^{|B| L_{1}} \mathrm{~d} P_{2} \times \sum_{N_{b}=0}^{|B| L_{1} L_{2} / 2 \pi} \quad \text { (charged mesons), }
$$


where $N_{b}$ labels the bound states as in the case without a magnetic field. These expressions will be used for the calculation of the partition function below.

\subsection{Mesonic partition function at finite temperature}

Finally we investigate the partition function at the temperature that is small enough to apply the dilute meson gas picture. At $B=0$, the non-interacting hadron resonance gas (HRG) picture reproduces the lattice data in a good accuracy until the temperature reaches the (pseudo)-critical temperature $T_{c}$. Around $T_{c}$, the thermally excited hadrons begin to overlap each other, and the interactions are no longer negligible; this is the regime where quarks and gluons start to emerge as the manifest microscopic degrees of freedom, leading to the quark-gluon plasma. We apply this established picture to the thermodynamics in the presence of large $B$.

Below we focus on the mesonic contribution to the pressure. At the dilute regime such that the interactions are negligible, the partition function for mesons is written as

$$
Z_{M}(T) \simeq \prod_{n} \prod_{P} \frac{1}{1-\mathrm{e}^{-E_{n}(P) / T}}
$$

where $n$ and $P$ label a bound state and its total momentum, respectively. The corresponding pressure $^{5}$ is

$$
\mathcal{P}_{M}(T) \simeq-T \sum_{n} \sum_{P} \ln \left(1-\mathrm{e}^{-E_{n}(P) / T}\right)
$$

where $E_{n}$ is the energy of a meson.

Now we use the specific forms of the density of states obtained in the previous subsection. Contributions from the neutral mesons made of the LLLs can be written as

$$
\mathcal{P}_{M}^{\text {neutral }}(T) \simeq-T \sum_{f, f^{\prime}} \delta_{e_{f}, e_{f^{\prime}}} \sum_{n_{3}} \int_{\vec{P}_{\perp}} \int_{P_{3}} \ln \left(1-\mathrm{e}^{-E_{n_{3}, \vec{P}_{\perp}}^{f f^{\prime}}\left(P_{3}\right) / T}\right)+\cdots
$$

where the ellipses denote contributions from the interactions and the hLLs, and $\delta_{e_{f}, e_{f^{\prime}}}$ is unity for equal charges, otherwise zero. We should not take the spin sum because the flavor and the direction of spin is locked in the LLL. As discussed in the last subsection, the integrals with respect

\footnotetext{
${ }^{5}$ In this paper by pressure we mean the negative of the free energy density. Precisely speaking, there are two schemes to define the pressure depending on how one changes the volume of the system [43]. The first scheme fixes the magnetic field density $B$ and the pressure is spatially isotropic, while the second scheme fixes the total number of magnetic flux leading to the spatial anisotropy in pressure [44]. In this paper we consider pressure in the first scheme.
} 
to the two transverse momenta have the symmetric form in case of the neutral mesons. On the other hand the charged mesons made of the LLLs provide the following contributions

$$
\mathcal{P}_{M}^{\text {charged }}(T) \simeq-T \sum_{f, f^{\prime}}\left(1-\delta_{e_{f}, e_{f^{\prime}}}\right) \sum_{n_{3}} \sum_{n_{\perp}} c_{f f^{\prime}} \frac{|B|}{2 \pi} \int_{P_{3}} \ln \left(1-\mathrm{e}^{-E_{n_{3}, n_{\perp}}^{f f^{\prime}}\left(P_{3}\right) / T}\right)+\cdots
$$

where $n_{\perp}$ labels the bound state in the transverse dynamics. Note that we could integrate over $P_{2}$ and get the factor $c_{f f^{\prime}}|B| / 2 \pi$ since the spectrum is independent of $P_{2}: c_{f f^{\prime}}$ is some number associated with the electric charges of the flavors $f$ and $f^{\prime}$.

Following from the analyses of the Schwinger-Dyson and Bethe-Salpeter equations, one can deduce approximate forms of the low energy meson spectra for long-range interaction models. We define the mass of ground state meson at each quantum number as

$$
M_{n_{3}}^{\text {neutral }} \equiv E_{n_{3}, \vec{P}_{\perp}=\overrightarrow{0}}^{f f^{\prime}}\left(P_{3}=0\right), \quad M_{n_{3}}^{\text {charged }} \equiv E_{n_{3}, n_{\perp}=0}^{f f^{\prime}}\left(P_{3}=0\right)
$$

where $P_{3}=0$ and the quantum numbers for the transverse direction take the minimal number, e.g., $P_{2}=0$. While we have not shown explicit numerical solutions of the energy levels on the right-hand sides, we showed that with these quantum numbers the dynamics is governed by the $(1+1)$-dimensional dynamics in the $x_{L}$-direction, and the meson spectra are independent of the magnitude of $B$. Now we increase $P_{3}$. Since the Poincare invariance should be maintained in the $x_{L}$-direction, the spectra are

$$
E_{n_{3}, \vec{P}_{\perp}=\overrightarrow{0}}^{f f^{\prime}}\left(P_{3}\right)=\sqrt{\left(M_{n_{3}}^{\text {neutral }}\right)^{2}+P_{3}^{2}}, \quad E_{n_{3}, n_{\perp}=0}^{f f^{\prime}}\left(P_{3}\right) \simeq \sqrt{\left(M_{n_{3}}^{\text {charged }}\right)^{2}+P_{3}^{2}} .
$$

Next we further increase the quantum numbers associated with the transverse dynamics. There is no kinetic term in the transverse directions, so that the contributions to the spectra arise only from the potential energy which we have examined approximately in Eqs. (48) and (74). Including them as corrections to the above spectra, we arrive at

$$
E_{n_{3}, \vec{P}_{\perp}}^{f f^{\prime}}\left(P_{3}\right) \simeq \sqrt{\left(M_{n_{3}}^{\text {neutral }}\right)^{2}+P_{3}^{2}}+c_{1} \Lambda_{\mathrm{QCD}}^{3} \frac{P_{\perp}^{2}}{|B|^{2}}+\cdots, \quad \text { (for neutral mesons) }
$$

and

$$
E_{n_{3}, n_{\perp}}^{f f^{\prime}}\left(P_{3}\right) \simeq \sqrt{\left(M_{n_{3}}^{\text {charged }}\right)^{2}+P_{3}^{2}}+c_{2} \Lambda_{\mathrm{QCD}}^{3} \frac{n_{\perp}}{|B|}+\cdots, \quad \text { (for charged mesons) }
$$

where $c_{1}$ and $c_{2}$ are dimensionless constant ${ }^{6}$.

\footnotetext{
${ }^{6}$ Strictly speaking, $c_{1}$ and $c_{2}$ can be functions of $P_{3}$ since our evaluation is based on the expansion of $\sim 1 /\left\langle r_{3}\right\rangle$ and $\left\langle r_{3}\right\rangle$ can depend on the Lorentz boost. But as far as $P_{3}$ is small we can treat $c_{1}$ and $c_{2}$ as constants and this is the situation we will discuss in the following.
} 
At temperature lower than the meson masses such that $T \ll M_{n_{3}}$, one can use the expansion $\ln \left(1-\mathrm{e}^{-x}\right) \simeq-\mathrm{e}^{-x}$ to obtain an analytic form of the pressure, and then finds that both of the contributions from neutral and charged mesons behave as

$$
\mathcal{P}_{n}(T) \sim T^{2} \frac{|B|^{2}}{\Lambda_{\mathrm{QCD}}^{3}} \int_{P_{3}} \mathrm{e}^{-\sqrt{M_{n_{3}}^{2}+P_{3}^{2}} / T} \sim T^{2} \frac{|B|^{2}}{\Lambda_{\mathrm{QCD}}^{3}} \times\left(M_{n_{3}} T\right)^{1 / 2} \mathrm{e}^{-M_{n_{3}} / T}+\cdots
$$

where we used the non-relativistic approximation to get the final expression, and a factor of $|B|^{2}$ is originated from the transverse phase space volume, namely either the integration of $P_{2}$ or summation of $n_{2}$. Since a lot of low energy states is involved in the transverse dynamics, we get a big phase factor $\sim|B|^{2} / \Lambda_{\mathrm{QCD}}^{2}$. The above should be compared with the pressure at $B=0$ generated by mesons with mass $M_{n}^{\prime}$,

$$
\mathcal{P}_{n}^{B=0}(T) \sim T \int_{\vec{P}} \mathrm{e}^{-\sqrt{M_{n}^{\prime 2}+\vec{P}^{2}} / T} \sim T \times\left(M_{n}^{\prime} T\right)^{3 / 2} \mathrm{e}^{-M_{n}^{\prime} / T}+\cdots
$$

In both $B=0$ and large $B$ cases, the pressures have the same temperature dependences. However, clearly, the magnetic-field dependence of the rest mass $M_{n}$ plays a crucial role to determine whether the effects of strong magnetic fields act to enhance or suppress the pressure $\mathcal{P}$. Recall that, for long-range interactions, the magnetic-field dependence decouples from $M_{n}$ at very large $|B|$, and $M_{n}$ stays around $\sim \Lambda_{\mathrm{QCD}}$. In this regime, the mesonic pressure keeps growing as $|B|$ increases. Then the system reaches the percolation region at smaller $T$ than in the $B=0$ case; namely, thermally excited hadrons overlap each other, so that gluons propagate from one to another hadron as if they are liberated from hadrons. The schematic picture is shown in Fig. 6 (left).

This percolation picture of meson gas can be also combined with the chiral restoration at finite $T$. The chiral condensate with the flavor $f$ can be calculated by taking a derivative of $\mathcal{P}$ with respect to the current quark mass as

$$
-\left\langle\bar{\psi}_{f} \psi_{f}\right\rangle_{T}=\frac{\partial \mathcal{P}_{\mathrm{vac}}}{\partial m_{f}}+\frac{\partial \mathcal{P}_{\text {excited }}}{\partial m_{f}} \simeq-\left\langle\bar{\psi}_{f} \psi_{f}\right\rangle_{T=0}-\sum_{n} \sum_{P} \frac{\partial E_{n}(P)}{\partial m_{f}} \frac{1}{\mathrm{e}^{E_{n}(P) / T}-1}
$$

where $\mathcal{P}_{\text {vac }}$ and $\mathcal{P}_{\text {excited }}$ are the contributions to pressure from the vacuum and thermally excited states, respectively, and $\partial E_{n} / \partial m_{f}$ is the sigma term of a meson. Note that the vacuum value of the condensate is negative $\langle\bar{\psi} \psi\rangle_{T=0}<0$, and also that the sigma term should be positive, giving a competing contribution to the vacuum contribution. Therefore, with more and more meson excitations, the chiral condensate is strongly diminished by the meson gas contributions.

We have already seen that the meson spectra at large $B$ stay as small as those in $B=0$, so that they are easily excited at finite temperature. While at very low temperature the vacuum contribution grows by the magnetic catalysis, the mesonic contribution at large $B$ grows faster than the vacuum contribution once they are activated in finite $T$, thanks to the large phase space 

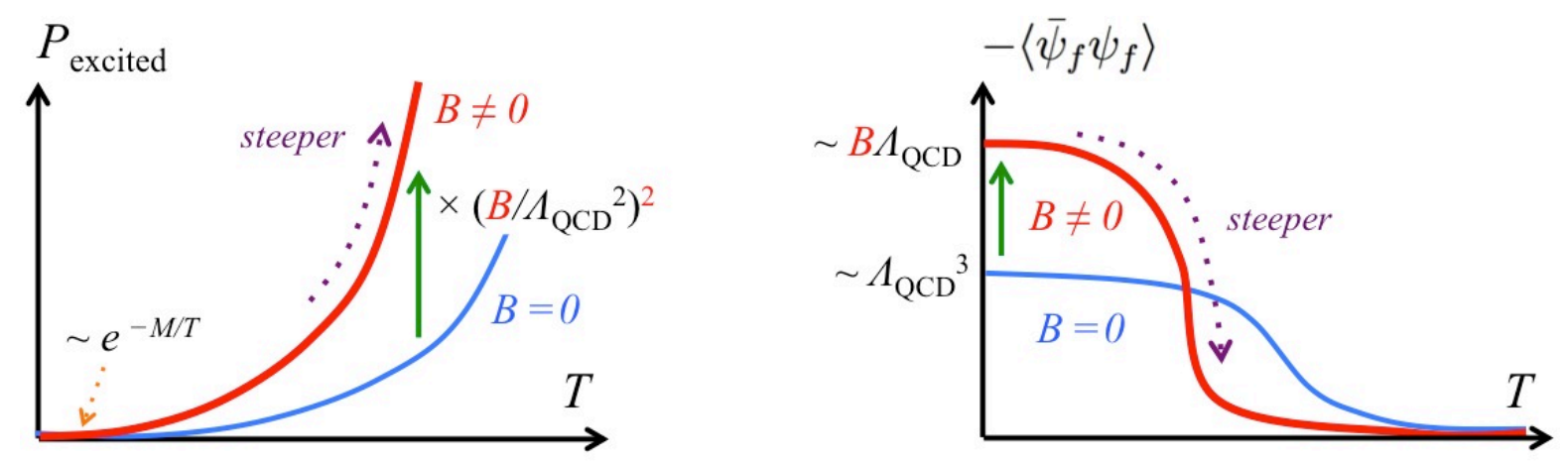

Figure 6: (Left) Contributions of the meson excitations to the finite $T$ pressure at $B=0$ and $B \neq 0 . P_{\text {excited }}$ at $|e B| \gg \Lambda_{\mathrm{QCD}}^{2}$ is bigger than the pressure at $B=0$ roughly by a factor of $\left(|e B| / \Lambda_{\mathrm{QCD}}^{2}\right)^{2}$. The pressure starts to contribute significantly when $T$ reaches $\sim M \sim \Lambda_{\mathrm{QCD}}$. (Right) The finite $T$ chiral condensate, $-\left\langle\bar{\psi}_{f} \psi_{f}\right\rangle_{T}$. At $T=0$, the (absolute value of) condensate grows linearly as $B$ increases. On the other hand, as $T$ increases, the condensate at $B \neq 0$ decreases more rapidly than the $B=0$ condensate because of the hadron resonances which emerge more radically at finite $B$, giving the chiral density of $\sim B^{2}$ with the opposite sign to the vacuum chiral condensate.

volume in the transverse dynamics. Therefore, the relative magnitude of the growth rates is inversed, leading to the dissociation of the chiral condensate at lower $T$ compared to the $B=0$ case [see Fig.6 (right)]. The percolation and chiral restoration are intimately connected; the enhanced number of hadronic excitations provides a simple explanation of the inverse magnetic catalysis phenomenon.

\section{Summary}

In this first part of a series of papers, we have provided heuristic arguments on the meson structures and spectra. Our analyses were specialized to models of long-range interactions such as the confining linear rising potential or harmonic oscillator. The key assumption was that these longrange contributions dominate over the short-range ones, although presumably this picture might oversimplify the realitity. Nevertheless, such studies can cover aspects of the interplay between nonperturbative QCD interactions and strong magnetic fields which have not been addressed in the previous studies since they primarily rely on effective models with short-range interactions.

On the basis of our assumption of the IR-dominant interaction, we systematically studied the parametric behaviors of the various quantities. These systematic results, covering from the constituent quark mass to the meson spectra and their thermodynamics, were missing in the conventional studies, and provides a consistent guideline to understand the lattice results. Especially, we argued an importance of the long-range interaction for understanding the lattice results and 
the reason why the conventional effective models of short-range interactions have not been able to explain them.

We note that, while there were several studies treating either the Schwinger-Dyson or BetheSalpeter equations at finite $B$ separately, our work is the first attempt to treat the quark selfenergies and meson spectra in a consistent way. The consistent treatments are essential when we consider quantities related to the spontaneous symmetry breaking, such as spectra of the Nambu-Goldstone bosons.

As found in our previous studies, the quark self-energies and mass gap are $B$-independent for interactions with the IR dominance. This behavior is in favor of explaining the linear $B$ dependence of the chiral condensate, which has been observed in the lattice simulations. With the same mechanism, it follows that the meson states at low energy are also nearly $B$-independent. Moreover, we found interesting $B$-dependence of the transverse momenta, which is essential to count the density of states of mesons at low energy. With this $B$-dependence of the meson spectra, it was shown that at low temperature, the pressure of meson gas parametrically grows as $\sim B^{2}$ as $|B|$ increases. This behavior was commonly found in contributions of both neutral and charged mesons. In particular, contributions from neutral mesons are drastically different from those at small $|B|$; strong magnetic fields can intrude a neutral composite meson, causing a strong structure change and modification of the spectrum.

The percolation and chiral restoration were discussed within the hadron resonance gas picture. Two phenomena are intimately connected by the overlap of thermally excited hadrons; at larger $B$, such overlap occurs at lower $T$, because of the enhanced phase space due to the magnetic fields.

Finally we close our discussions by repeating the importance of the IR gluons, since their impacts on the QCD phenomenology are so distinct from those based on perturbative gluons or short-range interactions. The examples can be found for QCD at finite density such as the quarkyonic chiral spirals [45, 46], at finite temperature such as the improved equation of state [47] and the massless mode which induces positivity violation [48], and also in external magnetic fields such as the quark mass gap which resolves the puzzle posed by the lattice results [12, 30]. Furthermore, the application of such an approach to heavy-ion phenomenology has shown signatures of a strongly coupled quark-gluon plasma [49]. All these studies so far have shown significant importance of the role of long-range interactions/confinement effects in various aspects of QCD under extreme conditions, ranging from static to dynamic properties. It is thus very encouraging to keep exploring along this direction, which will certainly improve our understanding of the QCD phase transition.

In the second part, we will address more quantitative aspects, using models of various types of interactions. 


\section{Acknowledgments}

This work was supported by NSF Grants PHY09-69790 and PHY13-05891 (T.K.), JSPS Grants-in-Aid No. 25287066 (K.H.), and the Helmholtz International Center for FAIR within the framework of the LOEWE program launched by the State of Hesse (N.S.).

\section{Appendix A. Dimensionally reduced operators}

In this paper we consider only the strong field regime where the LLL approximation is the proper starting point. In such a regime, usual classifications of states, in terms of the angular momentum or isospin, are not as useful as in the $B=0$ case. On the other hand, we are also interested in how meson states at $B=0$ are connected to those at large $B$. To observe the relation between these two limiting cases, we investigate how the meson interpolating fields at $B=0$ reduce to the $(1+1)$ dimensional fields in the LLLs. The good summary of the algebra can be also found in Ref.[50].

Appendix A.1. Quantum numbers of the LLL fields

Let us begin with the $\gamma_{5}$-operator,

$$
\gamma_{5}=\gamma_{0} \gamma_{1} \gamma_{2} \gamma_{3}=\left(-\mathrm{i} \gamma_{0} \gamma_{3}\right)\left(\mathrm{i} \gamma_{1} \gamma_{2}\right) \equiv \Gamma_{5} \sigma_{3}
$$

where $\sigma_{3}$ is the third component of the spin operator, and $\Gamma_{5} \equiv-\mathrm{i} \gamma_{0} \gamma_{3}$ can be regarded as the two-dimensional $\gamma_{5}^{2 \mathrm{D}}$ which characterizes the moving directions in the $x_{3}$-direction. Using $\Gamma_{5}$, we can define the right- and left-movers whose momenta are oriented in the $+x_{3}$ and $-x_{3}$ directions, respectively:

$$
\frac{1 \pm \Gamma_{5}}{2} \psi \equiv \psi_{r, l}
$$

We use the indices $(r, l)$ to label the moving directions (or two-dimensional chirality), and distinguish them from the four-dimensional chirality $(R, L)$. The relation between $(R, L)$ and $(r, l)$ depends on the spin directions of fields. We find the relations for spin up and down states, respectively, as

$$
\left(\psi_{\uparrow}\right)_{R, L}=\frac{1 \pm \gamma_{5}}{2} \psi_{\uparrow}=\frac{1 \pm \Gamma_{5}}{2} \psi_{\uparrow}=\left(\psi_{\uparrow}\right)_{r, l}, \quad\left(\psi_{\downarrow}\right)_{R, L}=\frac{1 \pm \gamma_{5}}{2} \psi_{\downarrow}=\frac{1 \mp \Gamma_{5}}{2} \psi_{\downarrow}=\left(\psi_{\downarrow}\right)_{l, r} .
$$

For the spin down states, the chiralities in four- and two-dimension appears to be opposite. 
Now let $B>0$ and consider the LLL fields. The spin-flavor contents are $\chi^{f}=\left(u_{\uparrow}, d_{\downarrow}, \cdots\right)$. As in the above, we find ${ }^{7}$

$$
\chi_{R}^{f}=\left(u_{\uparrow}^{r}, d_{\downarrow}^{l}, \cdots\right), \quad \chi_{L}^{f}=\left(u_{\uparrow}^{l}, d_{\downarrow}^{r}, \cdots\right) .
$$

When considering $B<0$, one should flip the direction of spin.

Appendix A.2. Quark bilinear operators

Next we map the four-dimensional quark bilinear operators from four to two space-time dimensions. In doing this we will keep only the LLL fields. The scalar and pseudo-scalar operators which can be made of the LLL fields are listed as

$$
(\bar{u} u, \bar{d} d)_{\mathrm{LLL}} \sim\left(\bar{\chi}_{u} \chi_{u}, \bar{\chi}_{d} \chi_{d}\right), \quad\left(\bar{u} \mathrm{i} \gamma_{5} u, \bar{d} \mathrm{i} \gamma_{5} d\right)_{\mathrm{LLL}} \sim\left(\bar{\chi}_{u} \mathrm{i} \Gamma_{5} \chi_{u},-\bar{\chi}_{d} \mathrm{i} \Gamma_{5} \chi_{d}\right)
$$

Operators such as a $\bar{u} d$ necessarily contain the hLL fields, so that such combinations were omitted here. These expressions imply that the scalar and pseudo-scalar mesons are light when they are neutral, e.g., $\pi^{0}$, and are as heavy as $\sqrt{|e B|}$ when they are charged, e.g., $\pi^{ \pm}$. Note that in the pseudo-scalar channel the sign of the operator flips for $d$-quarks. Due to this sign flip, the isosinglet (isovector) operators made of the LLLs in 4D behave as isovector (isosinglet) in 2D. This observation becomes important when one considers the annihilation of a quark and anti-quark in the LLLs into purely gluonic configurations, such as instantons.

For vector currents, there are two types of operators that couple to low energy states. The first type is composed of like-charge fields

$$
\left(\bar{u} \gamma_{L} u, \bar{d} \gamma_{L} d\right)_{\mathrm{LLL}} \sim\left(\bar{\chi}_{u} \gamma_{L} \chi_{u}, \bar{\chi}_{d} \gamma_{L} \chi_{d}\right)
$$

where $\gamma_{L}$ preserves the spin of fields. For instance, it couples $\bar{u}_{\uparrow}$ to $u_{\uparrow}$, or $\bar{d}_{\downarrow}$ to $s_{\downarrow}$, etc. The expression implies that vector mesons with $s_{z}=0$ become light when they are neutral, e.g., $\rho_{s_{z}=0}^{0}$ and $\omega_{s_{z}=0}$, but becomes heavy when they are charged, e.g., $\rho_{s_{z}=0}^{ \pm}$. The second type is composed of unlike-charge fields

$$
\left(\bar{u} \gamma_{\perp} d, \bar{d} \gamma_{\perp} u\right)_{\mathrm{LLL}} \sim\left(\bar{\chi}_{u} \gamma_{\perp} \chi_{d}, \bar{\chi}_{d} \gamma_{\perp} \chi_{u}\right)
$$

where $\gamma_{\perp}$ flips the spin of fields and thereby can couple the LLL fields with different flavors. In this respect, $\gamma_{\perp}$-matrices may be regarded as "flavor"-matrices for the LLL dynamics. Note also

\footnotetext{
${ }^{7}$ These expressions are useful to intuitively understand the charge separation effect in the presence of chiral imbalance. With an excess of the right-handed chirality, we have more $u_{\uparrow}$ quark moving in the $x_{3}$-direction, and more $d_{\downarrow}$ quark moving in the $-x_{3}$-direction.
} 
that $u$ and $d$ in the LLL have the opposite two-dimensional chirality, so that $\bar{\chi}_{u} \gamma_{\perp} \chi_{d}$ and $\bar{\chi}_{d} \gamma_{\perp} \chi_{u}$ behave as $2 \mathrm{D}$ scalar operators, rather than vector ones. Therefore $4 \mathrm{D}$ operators producing mesons with $s_{z}= \pm 1$ states end up with scalar mesons in $2 \mathrm{D}$ at large $B$. The expression implies the spin-charge locked states which are realized in such a way that $\rho_{s_{z}= \pm 1}^{ \pm}$are light, while $\rho_{s_{z}=\mp 1}^{ \pm}$, $\rho_{s_{z}= \pm 1}^{0}$, etc. are heavy.

Next we consider axial-vector currents. The longitudinal components can be related to the vector currents as

$$
\left(\bar{u} \gamma_{L} \gamma_{5} u, \bar{d} \gamma_{L} \gamma_{5} d\right)_{\mathrm{LLL}} \sim\left(\bar{\chi}_{u} \gamma_{L} \Gamma_{5} \chi_{u},-\bar{\chi}_{d} \gamma_{L} \Gamma_{5} \chi_{d}\right) \sim(-\mathrm{i}) \epsilon_{L L^{\prime}}\left(\bar{\chi}_{u} \gamma_{L^{\prime}} \chi_{u},-\bar{\chi}_{d} \gamma_{L^{\prime}} \chi_{d}\right)
$$

Therefore the 4D axial-vector and vector currents couple to the same $2 \mathrm{D}$ states. Since the $4 \mathrm{D}$ axial-vector currents also couple to the Nambu-Goldstone bosons, at large $|B|$ the correlators of axial-vector, vector, and pseudo-scalar show the same low energy behaviors governed by the LLLs. The transverse components are

$$
\left(\bar{u} \gamma_{\perp} \gamma_{5} d, \bar{d} \gamma_{\perp} \gamma_{5} u\right)_{\mathrm{LLL}} \sim\left(\bar{\chi}_{u} \gamma_{\perp} \Gamma_{5} \chi_{d},-\bar{\chi}_{d} \gamma_{\perp} \Gamma_{5} \chi_{u}\right)
$$

which can be regarded as flavor off-diagonal 2D pseudo-scalar operators.

For tensor operators, there are three kinds of operators. The first two operators do not mix up different spin states and can be reduced to $\left(\sigma_{\mu \nu}=\mathrm{i}\left[\gamma_{\mu}, \gamma_{\nu}\right] / 2\right)$

$$
\left(\bar{u} \sigma_{L L^{\prime}} u, \bar{d} \sigma_{L L^{\prime}} d\right)_{\mathrm{LLL}} \sim-\epsilon_{L L^{\prime}}\left(\bar{\chi}_{u} \Gamma_{5} \chi_{u}, \bar{\chi}_{d} \Gamma_{5} \chi_{d}\right)
$$

and

$$
\left(\bar{u} \sigma_{\perp \perp^{\prime}} u, \bar{d} \sigma_{\perp \perp^{\prime}} d\right)_{\mathrm{LLL}} \sim \epsilon_{\perp \perp^{\prime}}\left(\bar{\chi}_{u} \chi_{u},-\bar{\chi}_{d} \chi_{d}\right)
$$

The third one mixes spins (or flavors),

$$
\left(\bar{u} \sigma_{L \perp} d, \bar{d} \sigma_{L \perp} u\right)_{\mathrm{LLL}} \sim \mathrm{i}\left(\bar{\chi}_{u} \gamma_{L} \gamma_{\perp} \chi_{d}, \bar{\chi}_{d} \gamma_{L} \gamma_{\perp} \chi_{u}\right)
$$

which can be regarded as flavor off-diagonal 2D vector operators.

In summary, many 4D operators, that couple to different meson states, show the same asymptotic behaviors, and can be reduced to 2D operators. In particular, operators having very different structures in four dimensions can look the same in the language of 2D operators made of the LLLs. For instance, we have seen that the axial-vector, vector, and pseudo-scalar operators in 4D couple to the same pseudo-scalar mesons in 2D. This implies that in strong magnetic fields these correlators at a long distance scale are saturated by the same asymptotic states.

[1] V. A. Miransky and I. A. Shovkovy, Phys. Rept. 576 (2015) 1 [arXiv:1503.00732 [hep-ph]]; J. O. Andersen, W. R. Naylor and A. Tranberg, arXiv:1411.7176 [hep-ph]; D. Kharzeev, K. Landsteiner, A. Schmitt and H. U. Yee, Lect. Notes Phys. 871 (2013) pp.1. 
[2] D. E. Kharzeev, L. D. McLerran and H. J. Warringa, Nucl. Phys. A 803 (2008) 227 [arXiv:0711.0950 [hep-ph]]; K. Fukushima, D. E. Kharzeev and H. J. Warringa, Phys. Rev. D 78 (2008) 074033 [arXiv:0808.3382 [hep-ph]]; K. Tuchin, Adv. High Energy Phys. 2013 (2013) 490495 [arXiv:1301.0099]; L. McLerran and V. Skokov, Nucl. Phys. A 929 (2014) 184 [arXiv:1305.0774 [hep-ph]].

[3] V. Skokov, A. Y. Illarionov and V. Toneev, Int. J. Mod. Phys. A 24 (2009) 5925 [arXiv:0907.1396 [nucl-th]]; A. Bzdak and V. Skokov, Phys. Lett. B 710 (2012) 171 [arXiv:1111.1949 [hep-ph]]; V. Voronyuk, V. D. Toneev, W. Cassing, E. L. Bratkovskaya, V. P. Konchakovski and S. A. Voloshin, Phys. Rev. C 83 (2011) 054911 [arXiv:1103.4239 [nucl-th]]; W. T. Deng and X. G. Huang, Phys. Rev. C 85 (2012) 044907 [arXiv:1201.5108 [nucl-th]].

[4] T. Vachaspati, Phys. Lett. B 265 (1991) 258; K. Enqvist and P. Olesen, Phys. Lett. B 319 (1993) 178 [hep-ph/9308270].

[5] P. V. Buividovich, M. N. Chernodub, E. V. Luschevskaya and M. I. Polikarpov, Phys. Lett. B 682 (2010) 484 [arXiv:0812.1740 [hep-lat]]; M. D'Elia and F. Negro, Phys. Rev. D 83 (2011) 114028 [arXiv:1103.2080 [hep-lat]]; G. S. Bali, F. Bruckmann, G. Endrödi, Z. Fodor, S. D. Katz and A. Schäfer, Phys. Rev. D 86 (2012) 071502 [arXiv:1206.4205 [hep-lat]].

[6] G. S. Bali, F. Bruckmann, G. Endrödi, Z. Fodor, S. D. Katz, S. Krieg, A. Schäfer and K. K. Szabo, JHEP 1202 (2012) 044 [arXiv:1111.4956 [hep-lat]].

[7] V. G. Bornyakov, P. V. Buividovich, N. Cundy, O. A. Kochetkov and A. Schäfer, Phys. Rev. D 90 (2014) 3, 034501 [arXiv:1312.5628 [hep-lat]].

[8] G. S. Bali, F. Bruckmann, G. Endrödi, F. Gruber and A. Schäfer, JHEP 1304 (2013) 130 [arXiv:1303.1328 [hep-lat]].

[9] C. Bonati, M. D'Elia, M. Mariti, M. Mesiti, F. Negro and F. Sanfilippo, Phys. Rev. D 89 (2014) 11, 114502 [arXiv:1403.6094 [hep-lat]].

[10] V. P. Gusynin, V. A. Miransky and I. A. Shovkovy, Phys. Lett. B 349, 477 (1995) [hep$\mathrm{ph} / 9412257]$.

[11] K. Fukushima, J. Phys. G 39 (2012) 013101 [arXiv:1108.2939 [hep-ph]].

[12] T. Kojo and N. Su, Phys. Lett. B 720 (2013) 192 [arXiv:1211.7318 [hep-ph]]; ibid. Nucl. Phys. A 931 (2014) 763 [arXiv:1407.7925 [hep-ph]]. 
[13] K. Hattori, K. Itakura, S. Ozaki and S. Yasui, Phys. Rev. D 92, 065003 (2015) [arXiv:1504.07619 [hep-ph]].

[14] S. Ozaki, K. Itakura and Y. Kuramoto, arXiv:1509.06966 [hep-ph].

[15] I. A. Shovkovy, Lect. Notes Phys. 871 (2013) 13 [arXiv:1207.5081 [hep-ph]]; H. Suganuma and T. Tatsumi, Annals Phys. 208 (1991) 470; T. D. Cohen, D. A. McGady and E. S. Werbos, Phys. Rev. C 76 (2007) 055201 [arXiv:0706.3208 [hep-ph]]; R. Gatto and M. Ruggieri, Phys. Rev. D 83 (2011) 034016 [arXiv:1012.1291 [hep-ph]]; J. O. Andersen, Phys. Rev. D 86 (2012) 025020 [arXiv:1202.2051 [hep-ph]]; J. O. Andersen, JHEP 1210 (2012) 005 [arXiv:1205.6978 [hep-ph]].

[16] G. Endrödi, JHEP 1507 (2015) 173 [arXiv:1504.08280 [hep-lat]].

[17] R. Gatto and M. Ruggieri, Phys. Rev. D 83 (2011) 034016 [arXiv:1012.1291 [hep-ph]]; A. J. Mizher, M. N. Chernodub and E. S. Fraga, Phys. Rev. D 82 (2010) 105016 [arXiv:1004.2712 [hep-ph]]; K. Kashiwa, Phys. Rev. D 83 (2011) 117901 [arXiv:1104.5167 [hep-ph]].

[18] K. Fukushima and Y. Hidaka, Phys. Rev. Lett. 110 (2013) 3, 031601 [arXiv:1209.1319 [hep$\mathrm{ph}]$.

[19] J. Chao, P. Chu and M. Huang, Phys. Rev. D 88 (2013) 054009 [arXiv:1305.1100 [hep-ph]]. L. Yu, H. Liu and M. Huang, Phys. Rev. D 90 (2014) 7, 074009 [arXiv:1404.6969 [hep-ph]]; L. Yu, J. Van Doorsselaere and M. Huang, Phys. Rev. D 91 (2015) 7, 074011 [arXiv:1411.7552 [hep-ph]].

[20] B. Feng, D. f. Hou and H. c. Ren, Phys. Rev. D 92 (2015) 6, 065011 [arXiv:1412.1647 [condmat.quant-gas]].

[21] F. Bruckmann, G. Endrödi and T. G. Kovacs, JHEP 1304 (2013) 112 [arXiv:1303.3972 [heplat]].

[22] E. J. Ferrer, V. de la Incera and X. J. Wen, Phys. Rev. D 91 (2015) 5, 054006 [arXiv:1407.3503 [nucl-th]].

[23] R. L. S. Farias, K. P. Gomes, G. I. Krein and M. B. Pinto, Phys. Rev. C 90 (2014) 2, 025203 [arXiv:1404.3931 [hep-ph]].

[24] M. Ferreira, P. Costa, O. Lourenço, T. Frederico and C. Providência, Phys. Rev. D 89 (2014) 11, 116011 [arXiv:1404.5577 [hep-ph]]. 
[25] N. Mueller and J. M. Pawlowski, Phys. Rev. D 91 (2015) 11, 116010 [arXiv:1502.08011 [hep$\mathrm{ph}]$.

[26] V. Skokov, Phys. Rev. D 85 (2012) 034026 [arXiv:1112.5137 [hep-ph]].

[27] K. Fukushima and J. M. Pawlowski, Phys. Rev. D 86 (2012) 076013 [arXiv:1203.4330 [hep$\mathrm{ph}]]$.

[28] K. Kamikado and T. Kanazawa, JHEP 1403 (2014) 009 [arXiv:1312.3124 [hep-ph]]; ibid. JHEP 1501 (2015) 129 [arXiv:1410.6253 [hep-ph]].

[29] S. Ozaki, Phys. Rev. D 89 (2014) 5, 054022 [arXiv:1311.3137 [hep-ph]]; S. Ozaki, T. Arai, K. Hattori and K. Itakura, Phys. Rev. D 92 (2015) 1, 016002 [arXiv:1504.07532 [hep-ph]].

[30] P. Watson and H. Reinhardt, Phys. Rev. D 89 (2014) 4, 045008 [arXiv:1310.6050 [hep-ph]]; N. Mueller, J. A. Bonnet and C. S. Fischer, Phys. Rev. D 89 (2014) 9, 094023 [arXiv:1401.1647 [hep-ph]].

[31] G. 't Hooft, Nucl. Phys. B 75 (1974) 461; C. G. Callan, Jr., N. Coote and D. J. Gross, Phys. Rev. D 13 (1976) 1649; I. Bars and M. B. Green, Phys. Rev. D 17 (1978) 537.

[32] M. N. Chernodub, J. Van Doorsselaere and H. Verschelde, Phys. Rev. D 85 (2012) 045002 [arXiv:1111.4401 [hep-ph]].

[33] Y. A. Simonov, B. O. Kerbikov and M. A. Andreichikov, arXiv:1210.0227 [hep-ph]. M. A. Andreichikov, B. O. Kerbikov, V. D. Orlovsky and Y. A. Simonov, Phys. Rev. D 87 (2013) 9, 094029 [arXiv:1304.2533 [hep-ph]].

[34] J. Alford and M. Strickland, Phys. Rev. D 88 (2013) 105017 [arXiv:1309.3003 [hep-ph]].

[35] H. Taya, Phys. Rev. D 92 (2015) 1, 014038 [arXiv:1412.6877 [hep-ph]].

[36] S. Cho, K. Hattori, S. H. Lee, K. Morita, and S. Ozaki, Phys. Rev. Lett. 113, 172301 (2014); ibid. Phys. Rev. D 91, 045025 (2015); P. Gubler, K. Hattori, S. H. Lee, M. Oka, S. Ozaki and K. Suzuki, arXiv:1512.08864 [hep-ph].

[37] Y. Hidaka and A. Yamamoto, Phys. Rev. D 87 (2013) 9, 094502 [arXiv:1209.0007 [hep-ph]].

[38] E. V. Luschevskaya, O. E. Solovjeva, O. A. Kochetkov and O. V. Teryaev, Nucl. Phys. B 898 (2015) 627 [arXiv:1411.4284 [hep-lat]].

[39] G. Endrödi, JHEP 1304 (2013) 023 [arXiv:1301.1307 [hep-ph]]. 
[40] N. O. Agasian and S. M. Fedorov, Phys. Lett. B 663 (2008) 445 [arXiv:0803.3156 [hep-ph]].

[41] V. D. Orlovsky and Y. A. Simonov, Phys. Rev. D 89 (2014) 5, 054012 [arXiv:1311.1087 [hep-ph]].

[42] T. Kojo and N. Su, Phys. Lett. B 726 (2013) 839 [arXiv:1305.4510 [hep-ph]].

[43] G. S. Bali, F. Bruckmann, G. Endrödi, S. D. Katz and A. Schäfer, JHEP 1408 (2014) 177 [arXiv:1406.0269 [hep-lat]].

[44] E. J. Ferrer and V. de la Incera, Lect. Notes Phys. 871 (2013) 399 [arXiv:1208.5179 [nucl-th]]; E. J. Ferrer, V. de la Incera, J. P. Keith, I. Portillo and P. L. Springsteen, Phys. Rev. C 82 (2010) 065802 [arXiv:1009.3521 [hep-ph]].

[45] T. Kojo, Y. Hidaka, L. McLerran and R. D. Pisarski, Nucl. Phys. A 843 (2010) 37 [arXiv:0912.3800 [hep-ph]]; T. Kojo, R. D. Pisarski and A. M. Tsvelik, Phys. Rev. D 82 (2010) 074015 [arXiv:1007.0248 [hep-ph]]; T. Kojo, Y. Hidaka, K. Fukushima, L. D. McLerran and R. D. Pisarski, Nucl. Phys. A 875 (2012) 94 [arXiv:1107.2124 [hep-ph]].

[46] E. J. Ferrer, V. de la Incera and A. Sanchez, Acta Phys. Polon. Supp. 5 (2012) 679 [arXiv:1205.4492 [nucl-th]].

[47] D. Zwanziger, Phys. Rev. Lett. 94 (2005) 182301 [hep-ph/0407103]; D. Zwanziger, Phys. Rev. D 76 (2007) 125014 [hep-ph/0610021]; K. Fukushima and N. Su, Phys. Rev. D 88 (2013) 076008 [arXiv:1304.8004 [hep-ph]]; F. E. Canfora, D. Dudal, I. F. Justo, P. Pais, L. Rosa and D. Vercauteren, Eur. Phys. J. C 75 (2015) 7, 326 [arXiv:1505.02287 [hep-th]].

[48] N. Su and K. Tywoniuk, Phys. Rev. Lett. 114 (2015) 16, 161601 [arXiv:1409.3203 [hep-ph]].

[49] M. Haas, L. Fister and J. M. Pawlowski, Phys. Rev. D 90 (2014) 091501 [arXiv:1308.4960 [hep-ph]]; N. Christiansen, M. Haas, J. M. Pawlowski and N. Strodthoff, Phys. Rev. Lett. 115 (2015) 11, 112002 [arXiv:1411.7986 [hep-ph]]; W. Florkowski, R. Ryblewski, N. Su and K. Tywoniuk, arXiv:1504.03176 [hep-ph]; W. Florkowski, R. Ryblewski, N. Su and K. Tywoniuk, arXiv:1509.01242 [hep-ph]; A. Bandyopadhyay, N. Haque, M. G. Mustafa and M. Strickland, arXiv:1508.06249 [hep-ph].

[50] G. Basar, G. V. Dunne and D. E. Kharzeev, Phys. Rev. Lett. 104 (2010) 232301 [arXiv:1003.3464 [hep-ph]]. 